\title{
Nonlinear elliptic equations with measure valued absorption potentials
}

\author{
Nicolas Saintier* Laurent Véron ${ }^{\dagger}$
}

\begin{abstract}
We study the semilinear elliptic equation $-\Delta u+g(u) \sigma=\mu$ with Dirichlet boundary conditions in a smooth bounded domain where $\sigma$ is a nonnegative Radon measure, $\mu$ a Radon measure and $g$ is an absorbing nonlinearity. We show that the problem is well posed if we assume that $\sigma$ belongs to some Morrey class. Under this condition we give a general existence result for any bounded measure provided $g$ satisfies a subcritical integral assumption. We study also the supercritical case when $g(r)=|r|^{q-1} r$, with $q>1$ and $\mu$ satisfies an absolute continuity condition expressed in terms of some capacities involving $\sigma$.
\end{abstract}

2010 Mathematics Subject Classification. 35 J 61; 31 B 15; 28 C 05 .

Key words: Radon measures; Morrey class; capacities; potential estimates; $\theta$-regular measures .

\section{Contents}

1 Introduction $r$

2 Preliminaries $\quad \mathbf{7}$

2.1 Morrey spaces of measures . . . . . . . . . . . . . . . . . . . 8

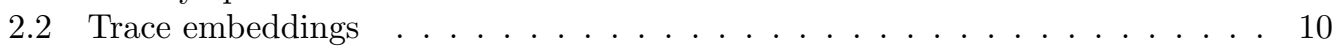

3 The subcritical case $\quad 12$

3.1 The variational construction . . . . . . . . . . . . . . . . . . . 12

3.2 The $L^{1}$ case . . . . . . . . . . . . . . . . . . . . . . . . . 15

3.3 Diffuse case .......................... . . . 23

3.4 Subcritical nonlinearities: proof of Theorem B. . . . . . . . . . . . . . . . . 24

4 The 2-D case $r$

*Departamento de Matemática, Facultad de Ciencias Exactas y Naturales, Universidad de Buenos Aires, Buenos Aires, Argentina.

Email: nsaintie@dm.uba.ar

†Département de Mathématiques, Université François Rabelais, Tours, France.

Email: veronl@lmpt.univ-tours.fr 
5 The supercritical case $\quad 31$

5.1 Proof of Theorem D ...................... . . . . . . . . . . . . . . . . . . .

5.2 Reduced measures ... . . . . . . . . . . . . . . . . . 33

5.3 The capacitary framework . . . . . . . . . . . . . . . . 36

5.4 The case $g(u)=|u|^{q-1} u$. . . . . . . . . . . . . . . . . . . . . . 38

5.5 Removable singularities ...................... 43

\section{Introduction}

Let $\Omega \subset \mathbb{R}^{N}$ be a bounded domain with a $C^{2}$ boundary, $\sigma$ a nonnegative Radon measure in $\Omega$ and $g: \mathbb{R} \rightarrow \mathbb{R}$ a continuous function satisfying, for some $r_{0} \geq 0$,

$$
r g(r) \geq 0 \quad \text { for all } r \in\left(-\infty,-r_{0}\right] \cup\left[r_{0}, \infty\right) .
$$

In this article we consider the following problem

$$
\begin{aligned}
-\Delta u+g(u) \sigma & =\mu & & \text { in } \Omega \\
u & =0 & & \text { in } \partial \Omega,
\end{aligned}
$$

where $\mu$ is a Radon measure defined in $\Omega$. By a solution we mean a function $u \in L^{1}(\Omega)$ such that $\rho g(u) \in L_{\sigma}^{1}(\Omega)$, where $\rho(x)=\operatorname{dist}(x, \partial \Omega)$ and $L_{\sigma}^{1}(\Omega)$ is the Lebesgue space of functions integrable with respect to $\sigma$, satisfying

$$
-\int_{\Omega} u \Delta \zeta d x+\int_{\Omega} g(u) \zeta d \sigma=\int_{\Omega} \zeta d \mu
$$

for all $\zeta \in W_{0}^{1, \infty}(\Omega)$ such that $\Delta \zeta \in L^{\infty}(\Omega)$. In the sequel, such a solution is called a very weak solution. A measure $\mu$ such that the problem admits a solution is called a good measure. We emphasize on the particular cases where $g(r)=|r|^{q-1} r$ with $q>0$, or $g(r)=e^{\alpha r}-1$ with $\alpha>0$ and $N=2$.

When $\sigma$ is a measure with constant positive density with respect to the Lebesgue measure in $\mathbb{R}^{N}$, this problem has been initiated by Brezis and Benilan [4], [5] who gave a general existence result for any bounded measure $\mu$ under an integrability condition of $g$ at infinity; their proof is based upon an a priori estimate of approximate solutions $u_{n}$ in Lorentz spaces $L^{q, \infty}(\Omega)$, yielding the uniform integrability of $g\left(u_{n}\right)$ and hence the pre-compactness in $L^{1}(\Omega)$.

If $g(r)=|r|^{q-1} r$, integrability condition is fufilled if and only if $0<q<\frac{N}{N-2}$ (any $q>0$ if $N=2$ ). In the 2 -dim case the integrability condition have been replaced by the exponential order of growth of $g$ in [27]. When $g(u)=|u|^{q-1} u$ with $q \geq \frac{N}{N-2}$ not any bounded measure is eligible for solving (1.2). In fact Baras and Pierre [3] proved that when $N>2$ and $q>1$, a bounded Radon measure $\mu$ is eligible if and only if it vanishes on Borel sets with $c_{2, q^{\prime}}$-capacity zero, where $q^{\prime}=\frac{q}{q-1}$ is the conjugate exponent of $q$. Contrary to the previous subcritical case, the method for proving the necessity of this condition is based upon a duality-convexity argument, while the sufficiency uses the fact that any positive Radon measure absolutely continuous with respect to the $c_{2, q^{\prime}}$-capacity can be approximated from below by an nondecreasing sequence of positive measures in $W^{-2, q}(\Omega)$ (see [13]). Furthermore they also 
gave a necessary and sufficient condition for a compact subset $K \subset \Omega$ to be removable for equation

$$
-\Delta u+|u|^{q-1} u=0 \quad \text { in } \Omega \backslash K,
$$

namely that $c_{2, q^{\prime}}(K)=0$.

The aim of this paper is to extend the previous constructions of Benilan-Brezis, BarasPierre and Vazquez to the case where $\sigma$ is a general measure. In order to be able to deal with the convergence of approximate solutions we assume that $\sigma$ belongs to the Morrey class $\mathcal{M}_{\frac{N}{N-\theta}}^{+}(\Omega)$ for some $\theta \in[0, N]$ which means

$$
\left|B_{r}(x)\right|_{\sigma}:=\int_{B_{r}(x)} d \sigma \leq c r^{\theta} \quad \text { for all }(x, r) \in \Omega \times(0, \infty)
$$

for some $c>0$. Note that we extend $\sigma$ by 0 in $\mathbb{R}^{N} \backslash \Omega$ and slightly abuse notation putting $\frac{N}{N-\theta}=\infty$ when $\theta=N$.

Our first result is the following:

Theorem A Assume $\sigma \in \mathcal{M}_{\frac{N}{N-\theta}}^{+}(\Omega)$ for some $\theta \in(N-2, N]$ and that $g$ satisfies (1.1). Then, for any $\mu \in L_{\rho}^{1}(\Omega)$, there exists a very weak solution u of problem (1.3). If we assume moreover that $g$ is nondecreasing and if $u^{\prime}$ is a very weak solution of (1.3) with right-hand side $\mu^{\prime} \in L_{\rho}^{1}(\Omega)$, then the following estimates hold

$$
-\int_{\Omega}\left|u-u^{\prime}\right| \Delta \zeta d x+\int_{\Omega}\left|g(u)-g\left(u^{\prime}\right)\right| \zeta d \sigma \leq \int_{\Omega}\left|\mu-\mu^{\prime}\right| d x
$$

and

$$
-\int_{\Omega}\left(u-u^{\prime}\right)_{+} \Delta \zeta d x+\int_{\Omega}\left(g(u)-g\left(u^{\prime}\right)\right)_{+} \zeta d \sigma \leq \int_{\Omega}\left(\mu-\mu^{\prime}\right)_{+} d x
$$

for all $\zeta \in W_{0}^{1, \infty}(\Omega)$ such that $\Delta \zeta \in L^{\infty}(\Omega)$ and $\zeta \geq 0$.

Note that (1.6) implies the uniqueness of the solution of (1.3), that we denote by $u_{\mu}$, and (1.7) the monotonicity of the mapping $\mu \mapsto u_{\mu}$.

The next result extends Benilan-Brezis unconditional existence result for measures.

Theorem B Let $N>2$ and $\sigma \in \mathcal{M}_{\frac{N}{N-\theta}}^{+}(\Omega)$ with $N \geq \theta>N-\frac{N}{N-1}$. Assume that $g$ satisfies (1.1) and $|g(r)| \leq \tilde{g}(|r|)$ for all $|r| \geq r_{0}$ where $\tilde{g}$ is a continuous nondecreasing function on $\left[r_{0}, \infty\right)$ verifying

$$
\int_{r_{0}}^{\infty} \tilde{g}(t) t^{-1-\frac{\theta}{N-2}} d t<\infty
$$


Then, for any bounded Radon measure $\mu$, there exists a very weak solution $u$ of problem (1.3) which moreover belongs to $L_{\sigma}^{1}(\Omega)$. Moreover, if we assume that $g$ is nondecreasing then the solution is unique.

Note that we recover Benilan-Brezis result when $\sigma$ is the Lebesgue measure (so that $\theta=N)$. Note also that when $g(r)=|r|^{q-1} r$, the integrability condition (1.8) is fullfilled if and only if $0<q<\frac{\theta}{N-2}$.

In the 2-dimensional case the condition on $\theta$ is $2 \geq \theta>0$ but (1.8) has to be modified. If $f: \mathbb{R} \mapsto \mathbb{R}_{+}$is nondecreasing we define its exponential order of growth at $\infty$ (see [27]) by

$$
a_{\infty}(f)=\inf \left\{\alpha \geq 0: \int_{0}^{\infty} f(s) e^{-\alpha s} d s<\infty\right\} .
$$

Similarly, if $h: \mathbb{R} \mapsto \mathbb{R}_{-}$is nondecreasing its exponential order of growth at $-\infty$ is

$$
a_{-\infty}(h)=\sup \left\{\alpha \leq 0: \int_{-\infty}^{0} h(s) e^{\alpha s} d s>-\infty\right\}
$$

If $g: \mathbb{R} \mapsto \mathbb{R}$ satisfies (1.1) but is not necessarily nondecreasing, we define the monotone nondecreasing hull $g^{*}$ of $g$ by

$$
g^{*}(r)= \begin{cases}\sup \{g(s): s \leq r\} & \text { for all } r \geq r_{0} \\ 0 & \text { for all } r \in\left(-r_{0}, r_{0}\right) \\ \inf \{g(s): s \geq r\} & \text { for all } r \leq-r_{0}\end{cases}
$$

We set

$$
a_{\infty}(g)=a_{\infty}\left(g_{+}^{*}\right) \text { and } a_{-\infty}(g)=a_{-\infty}\left(g_{-}^{*}\right)
$$

Theorem C Let $\sigma \in \mathcal{M}_{\frac{2}{2-\theta}}^{+}(\Omega)$ with $2 \geq \theta>0$ and $g: \mathbb{R} \mapsto \mathbb{R}$ satisfies (1.1).

(I) If $a_{\infty}(g)=0=a_{-\infty}(g)$, then for any $\mu \in \mathfrak{M}_{b}(\Omega)$, problem (1.3) admits a very weak solution.

(II) If $0<a_{\infty}(g)<\infty$ and $-\infty<a_{-\infty}(g)<0$ there exists $\delta>0$ such that if $\mu \in \mathfrak{M}_{b}(\Omega)$ satisfies $\|\mu\|_{\mathfrak{M}_{b}} \leq \delta$ problem (1.3) admits a very weak solution.

In the supercritical case, that is when (1.8) is not satisfied, all the measures are not eligible for solving (1.3). Following [16], [28, Th 4.2 ] we can give a sufficient existence condition involving the Green function of the Laplacian. Let $G(.,$.$) be the$ Green kernel defined in $\Omega \times \Omega$ and $\mathbb{G}[$.$] the corresponding potential operator acting$ on bounded measures $\nu$ namely $\mathbb{G}[\nu](x)=\int_{\Omega} G(x, y) d \nu(y)$. We have the following result: 
Theorem D Let $\sigma \in \mathcal{M}_{\frac{N}{N-\theta}}^{+}(\Omega)$ with $N \geq \theta>N-\frac{N}{N-1}$ and assume that $g$ is nondecreasing and vanishes at 0 .

(I) If $\mu \in \mathfrak{M}_{b}(\Omega)$ satisfies

$$
\rho g(\mathbb{G}[|\mu|]) \in L_{\sigma}^{1}(\Omega),
$$

then problem (1.3) admits a unique very weak solution.

(II) Let $\mu=\mu_{r}+\mu_{s}$ where $\mu_{r}$ is absolutely continuous with respect to the Lebesgue measure and $\mu_{s}$ is singular. Assume that $g$ satisfies the $\Delta_{2}$ condition, namely that

$$
\left|g\left(r+r^{\prime}\right)\right| \leq a\left(|g(r)|+\left|g\left(r^{\prime}\right)\right|\right)+b \quad \text { for all } r, r^{\prime} \in \mathbb{R},
$$

for some $a>1$ and $b \geq 0$. Then the previous assertion holds if (1.13) is replaced by

$$
\rho g\left(\mathbb{G}\left[\left|\mu_{s}\right|\right]\right) \in L_{\sigma}^{1}(\Omega) .
$$

Notice that (1.13) holds if either (i) $\sigma$ and $\mu$ have disjoint support, or (ii) $\mu \in \mathcal{M}_{p}(\Omega)$ for some $p>\frac{N}{2}$. Indeed if (i) holds then $\mathbb{G}[|\mu|]$ is bounded pointwise on the support of $\sigma$, and if (ii) holds then by Lemma $2.2 \mathbb{G}[|\mu|]$ is bounded pointwise in $\Omega$. Obviously the same comment holds in the setting of II.

In order to make more explicit conditions (1.13), (1.15), we introduce the following growth assumption on $g$ :

$$
|g(r)| \leq c\left(1+|r|^{q}\right) \quad \text { for all } r \in \mathbb{R},
$$

for some $q>1$. Notice that $\tilde{g}(r)=1+r^{q}$ satisfies (1.8) if and only if $q<\frac{\theta}{N-2}$. When $\sigma$ is the Lebesgue measure and $g(r)=|r|^{q-1} r$, Baras and Pierre [3] gave a necessary and sufficient condition for the existence of a solution to (1.2) involving certain capacities associated to the Bessel potential spaces $H^{s, p}\left(\mathbb{R}^{N}\right)$ where $s \in \mathbb{R}$ and $p \in[1, \infty]$. Let us recall that

$$
H^{s, p}\left(\mathbb{R}^{N}\right)=\left\{f: f=\mathbf{G}_{s} * h, h \in L^{p}\left(\mathbb{R}^{N}\right)\right\},
$$

where $\mathbf{G}_{s}$ is the Bessel kernel of order $s$. By extension $\mathbf{G}_{0}=\delta_{0}$, hence $H^{s, p}\left(\mathbb{R}^{N}\right)=$ $L^{p}\left(\mathbb{R}^{N}\right)$. When $s$ is a positive integer, it is proved by Calderón [2, Theorem 1.2.3] that $H^{s, p}\left(\mathbb{R}^{N}\right)$ is the standard Sobolev space $W^{s, p}\left(\mathbb{R}^{N}\right)$. If $s>0$, we denote by $c_{s, p}$ the associated capacity, called the Bessel capacity. It is defined for any compact set $K \subset \mathbb{R}^{N}$ by

$$
c_{s, p}(K)=\inf \left\{\|\phi\|_{H^{s, p}}^{p}: \phi \in \mathcal{S}\left(\mathbb{R}^{N}\right), \phi \geq 1 \text { on } K\right\} .
$$

The definition of $c_{s, p}$ is then extended first to open sets and then to arbitrary sets. We refer to [2] for general properties of Bessel spaces and their associated capacities 
$c_{s, p}$. We say that a measure $\mu \in \mathfrak{M}_{b}(\Omega)$ is absolutely continuous with respect to the $c_{s, p^{-}}$-capacity if for any Borel subset $E \subset \mathbb{R}^{N}$,

$$
c_{s, p}(E)=0 \Longrightarrow|\mu|(E)=0 .
$$

Baras and Pierre's result states that equation (1.2), with $\sigma$ standing for the Lebesgue measure and $g(r)=|r|^{q-1} r$, has a solution if and only if $\mu$ is absolutely continuous with respect to the $c_{2, q^{\prime}}$-capacity. The next result generalizes the "if" part to the case where $\sigma$ belongs to some Morrey space.

Theorem E Let $\sigma \in \mathcal{M}_{\frac{N}{N-\theta}}^{+}(\Omega)$ with $N \geq \theta>N-\frac{N}{N-1}$ and assume that $g$ is nondecreasing and satisfies (1.1) and (1.16). Let $p>1$ and $s \geq 0$ such that $N>$ $s p>N-\theta$ and $\frac{\theta p}{N-s p} \geq q$. If $\mu \in \mathfrak{M}_{b}(\Omega)$ is absolutely continuous with respect to the

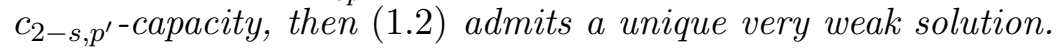

As a particular case, we take $p=q$ and obtain that if $\mu$ is absolutely continuous with respect to the $c_{2-\frac{N-\theta}{q}, q^{\prime}}$-capacity, then (1.3) admits a unique solution. We thus recover Baras-Pierre's sufficient condition [3] when $\theta=N$.

We give an explicit condition on the measure $\mu$ in terms of Morrey spaces implying that it satisfies the conditions of Theorem E.

Proposition 1.1 Under the assumptions on $\sigma$ and $g$ of Theorem $E$, if $\mu \in \mathcal{M}_{\frac{N}{N-\theta^{*}}}$

for some $\theta^{*}>\frac{(N-2) q-\theta}{q-1}$, then (1.3) admits a unique very weak solution.

Notice that the condition on $\mu$ given in Proposition 1.1 is weaker than the one given after Theorem D.

When $g(r)=|r|^{q-1} r$ with $q>1$, one can find a necessary conditions for the existence of a solution of (1.3) in the supercritical case under additional regularity assumptions on $\sigma$. By [2, Def 2.3.3, Prop. 2.3.5], the following expression

$$
c_{q}^{\sigma}(E)=\inf \left\{\int_{\Omega}|v|^{q^{\prime}} d \sigma: v \in L_{\sigma}^{q^{\prime}}(\Omega), v \geq 0, \mathbb{G}[v \sigma] \geq 1 \text { on } E\right\},
$$

where $E$ is any subset of $\Omega$ defines an outer capacity. The measure is called $\theta$-regular if

$$
\frac{1}{c} r^{\theta} \leq \int_{B_{r}(x)} d \sigma \leq c r^{\theta} \quad \text { for all }(x, r) \in \Omega \times(0,1],
$$

The next result gives a necessary condition for a measure to be a good measure.

Theorem F Let $q>1$ and $\sigma \in \mathcal{M}_{\frac{N}{N-\theta}}^{+}(\Omega)$ be $\theta$-regular with $N \geq \theta>N-2$. If $\mu \in \mathfrak{M}_{b}^{+}(\Omega)$ is such that problem (1.3) with $g(r)=|r|^{q-1} r$ admits a very weak solution, then $\mu$ vanishes on any Borel set $E$ such that $c_{q}^{\sigma}(E)=0$. 
Furthermore the $c_{q}^{\sigma}$ - capacity admits the following representation in terms of Besov capacities. If $\Gamma \subset \Omega$ is the support of $\sigma$, we denote by $B_{q^{\prime}, \infty}^{2-\frac{N-\theta}{q}, \Gamma}(\Omega)$ the closed subspace of distributions $\zeta \in B_{q^{\prime}, \infty}^{2-\frac{N-\theta}{q}}(\Omega)$ such that the support of the distribution $\Delta \zeta$ is a subset of $\Gamma$. Then

$$
c_{q}^{\sigma}(K) \sim c_{q^{\prime}, \infty}^{2-\frac{N-\theta}{q}, \Gamma}(K):=\inf \left\{\|\zeta\|_{B_{q^{\prime}, \infty}^{2-\frac{N-\theta}{q}}}^{q^{\prime}}: \zeta \in B_{q^{\prime}, \infty}^{2-\frac{N-\theta}{q}, \Gamma}(\Omega), \zeta \geq \chi_{K}\right\},
$$

for all compact subset $K \subset \Omega$.

Finally a complete characterization of removable sets can be obtained under a much stronger assumption on $\sigma$, namely that $d \sigma=w d x$ with $\omega:=w^{-\frac{1}{q-1}} \in L_{l o c}^{1}(\Omega)$. If $K \subset \Omega$ is compact, we set

$$
c_{q}^{\omega}(K)=\inf \left\{\int_{\Omega}|\Delta \zeta|^{q^{\prime}} \omega d x: \zeta \in C_{0}^{\infty}(\Omega), 0 \leq \zeta \leq 1, \zeta=1 \text { in a neighborhood of } K\right\} .
$$

This defines a capacity on Borel sets of $\Omega$.

Theorem G. Assume $q>1$ and there exists a nonnegative Borel function $w$ in $\Omega$ in the Muckenhoupt class $A_{q}(\Omega)$ such that $d \sigma=w d x$. If $K \subset \Omega$ is compact, a function $u \in L_{l o c}^{1}(\Omega \backslash K)$ such that $|u|^{q} w \in L_{l o c}^{1}(\Omega \backslash K)$ which satisfies

$$
-\Delta u+w|u|^{q-1} u=0,
$$

in the sense of distributions in $\Omega \backslash K$ can be extended as a solution of the same equation in the whole $\Omega$ if and only if $c_{q, w}(K)=0$.

The assumption $w \in A_{q}(\Omega)$ can be weakened and replaced by $\omega=w^{\frac{1}{1-q}}$ is $q^{\prime}$ admissible in the sense of [15, Chap 1], a condition which implies in particular the validity of the Gagliardo-Nirenberg and the Poincaré inequalities.

\section{Preliminaries}

In the whole paper $c$ denotes a generic positive constant whose value can change from one ocurrence to another even within a single string of estimates. Sometimes, in order to avoid ambiguity, we are led to introduce other notations for constant, for example $c^{\prime}$.

We denote by $\mathfrak{M}_{b}(\Omega)$ the space of outer regular bounded Borel measures on $\Omega$ equipped with the total variation norm, and by $\mathfrak{M}_{b}^{+}(\Omega)$ its positive cone. Since $\Omega$ is 
bounded we can identify bounded Radon measures in $\Omega$ with measures $\mu$ in $\bar{\Omega}$ such that $|\mu|(\partial \Omega)=0$. All the measures are extended by 0 in $\mathbb{R}^{N} \backslash \Omega$.

Let $G(.,$.$) be the Green kernel defined in \Omega \times \Omega$ and $\mathbb{G}[$.$] the corresponding$ potential operator acting on bounded measures $\nu$ namely $\mathbb{G}[\nu](x)=\int_{\Omega} G(x, y) d \nu(y)$. We denote $L^{p, \infty}(\Omega)$ the usual weak $L^{p}$ space. The next result is classical and valid in a much more general setting (see e.g. [6], [11]).

Lemma 2.1 Let $\mu \in \mathfrak{M}_{b}(\Omega)$ and $v=\mathbb{G}[\mu]$ be the (very weak) solution of

$$
\begin{aligned}
-\Delta v & =\mu & & \text { in } \Omega \\
v & =0 & & \text { in } \partial \Omega .
\end{aligned}
$$

I- If $N \geq 2$, then $v \in L^{\frac{N}{N-2}, \infty}(\Omega), \nabla v \in L^{\frac{N}{N-1}, \infty}(\Omega)$ and

$$
\|v\|_{L^{\frac{N}{N-2}}, \infty}+\|\nabla v\|_{L^{\frac{N}{N-1}, \infty}} \leq c\|\mu\|_{\mathfrak{M}_{b}} .
$$

II- If $N=2$, then $v \in B M O(\Omega), \nabla v \in L^{2, \infty}(\Omega)$ and

$$
\|v\|_{B M O}+\|\nabla v\|_{L^{2, \infty}} \leq c\|\mu\|_{\mathfrak{M}_{b}} .
$$

This result can be refined when more information is available on the degree of concentration of $\mu$. This leads to the definition of Morrey spaces of measures.

\subsection{Morrey spaces of measures}

If $1 \leq p \leq \infty$ we define the Morrey space $\mathcal{M}_{p}(\Omega)$ as the set of bounded outer regular Borel measures $\mu$ defined in $\Omega$ and extended by 0 in $\Omega^{c}$, satisfying

$$
\left|B_{r}(x)\right|_{\mu}:=\int_{B_{r}(x)} d|\mu| \leq c r^{N\left(1-\frac{1}{p}\right)} \quad \text { for all }(x, r) \in \Omega \times \mathbb{R}_{+},
$$

for some $c>0$. In particular $\mu \in \mathcal{M}_{\frac{N}{N-\theta}}(\Omega), \theta \in[0, N]$, if

$$
\int_{B_{r}(x)} d|\mu| \leq c r^{\theta} \quad \text { for all }(x, r) \in \Omega \times \mathbb{R}_{+} .
$$

We refer to [19] for a detailed study of $\mathcal{M}_{p}(\Omega)$ and full proofs of the various results we will recall now. Endowed with the norm

$$
\|\mu\|_{\mathcal{M}_{p}}=\sup _{(x, r) \in \Omega \times \mathbb{R}_{+}} r^{N\left(\frac{1}{p}-1\right)}\left|B_{r}(x)\right|_{\mu},
$$


$\mathcal{M}_{p}(\Omega)$ is a Banach space and $\mathcal{M}_{p}^{+}(\Omega)=\mathcal{M}_{p}(\Omega) \cap \mathfrak{M}_{b}^{+}(\Omega)$ is its positive cone. We also set $M_{p}(\Omega)=\mathcal{M}_{p}(\Omega) \cap L_{\text {loc }}^{1}(\Omega)$; it is a closed subspace of $\mathcal{M}_{p}(\Omega)$ and, if $1<p<\infty$, the following imbedding holds

$$
L^{p}(\Omega) \hookrightarrow L^{p, \infty}(\Omega) \hookrightarrow M_{p}(\Omega) .
$$

Note that since $\Omega$ is bounded and any measure in $\Omega$ is extended to $\mathbb{R}^{N}$ by 0 , it is easily seen that if $1 \leq q \leq p \leq \infty$ we have a continuous embedding $\mathcal{M}_{p}(\Omega) \hookrightarrow \mathcal{M}_{q}(\Omega)$ with

$$
\|v\|_{\mathcal{M}_{q}} \leq(\operatorname{diam}(\Omega))^{\frac{N}{q}-\frac{N}{p}}\|v\|_{\mathcal{M}_{p}} \quad \text { for all } v \in \mathcal{M}_{p}(\Omega) .
$$

Indeed for any $x \in \Omega$ the ball centered at $x$ with radius $\operatorname{diam}(\Omega)$ contains $\Omega$ so that it is enough to consider $r \leq \operatorname{diam}(\Omega)$. We have

$$
r^{-N(1-1 / q)}\left|B_{r}(x)\right|_{\mu} \leq r^{-N(1-1 / q)}\|\mu\|_{\mathcal{M}_{p}} r^{N(1-1 / p)} \leq(\operatorname{diam}(\Omega))^{\frac{N}{q}-\frac{N}{p}}\|\mu\|_{\mathcal{M}_{p}} .
$$

The following imbedding inequalities holds.

Lemma 2.2 Let $\mu \in \mathcal{M}_{p}(\Omega)$ and $v$ be the solution of (2.1).

I- If $1<p<\frac{N}{2}$, then $v \in M_{q}(\Omega)$ with $\frac{1}{q}=\frac{1}{p}-\frac{2}{N}$ and there holds

$$
\|v\|_{\mathcal{M}_{q}} \leq c\|\mu\|_{\mathcal{M}_{p}}
$$

II- If $p>\frac{N}{2}$, then $v$ is bounded pointwise and

$$
\begin{aligned}
& v(x) \leq c\|\mu\|_{\mathcal{M}_{p}} \quad \text { for all } x \in \Omega, \\
& \sup _{x \neq y} \frac{|v(x)-v(y)|}{|x-y|^{\alpha}} \leq c\|\mu\|_{\mathcal{M}_{p}} \text { with } \alpha=2-\frac{N}{p} \quad \text { if } \quad N>p>\frac{N}{2} \text {, } \\
& \sup _{x \neq y} \frac{|v(x)-v(y)|}{|x-y|^{\alpha}} \leq c\|\mu\|_{\mathcal{M}_{p}} \text { with } \alpha \in(0,1) \quad \text { if } N=p \text {, } \\
& \sup _{x}|\nabla v(x)| \leq c\|\mu\|_{\mathcal{M}_{p}} \quad \text { if } N<p .
\end{aligned}
$$

Remark. The previous regularity results are proved in [19, Prop. 3.1, 3.5] when $v=I_{\alpha} * \mu$ where $I_{\alpha}$ is the Riesz potential. However it is easily seen that the proof in [19] can be adapted to our setting. In particular for (2.8) we need that $G(x, y) \leq c|x-y|^{2-N}$, for (i) we use $(2.7)$.

Remark. If we assume that $\mu \in \mathfrak{M}_{\rho}(\Omega) \cap \mathcal{M}_{p, l o c}(\Omega)$, the previous estimates acquire a local aspect and remain valid provided the supremum in the norms on the left-hand sides are taken on compact subsets of $\Omega$. 


\subsection{Trace embeddings}

Some applications of Morrey spaces to imbedding theorems (also called trace inequalities) can be found in Adams-Hedberg's book [2]. For the sake of completeness, we quote here the main result therein we will use in the sequel. If $0<\alpha<N$ we recall that $I_{\alpha}$ (resp. $G_{\alpha}$ ) is the Riesz potential (resp. the Bessel potential) of order $\alpha$ in $\mathbb{R}^{N}$. The next result is $\left[2\right.$, Th 7.2.2, 7.3.2 ] (recall that the $c_{I_{\alpha}, p}$-Riesz capacity of a ball $B_{r}(x)$ is proportional to $r^{N-\alpha p}$ - see [2, Prop. 5.1.2].)

Proposition 2.3 Let $\sigma$ be a nonnegative Radon measure in $\mathbb{R}^{N}, N>\alpha p$ and $1<p<q<\frac{N p}{N-\alpha p}$.

(I)- The following assertions are equivalent:

$$
\left\|I_{\alpha} * f\right\|_{L_{\sigma}^{q}\left(\mathbb{R}^{N}\right)} \leq c_{1}\|f\|_{L^{p}\left(\mathbb{R}^{N}\right)} \quad \text { for all } f \in L^{p}\left(\mathbb{R}^{N}\right),
$$

for some $c_{1}=c_{1}(N, \alpha, p, q)>0$, and

$$
\sigma \in \mathcal{M}_{r}\left(\mathbb{R}^{N}\right) \quad \text { with } \frac{1}{r}=q\left(\frac{1}{q}-\frac{1}{p}+\frac{\alpha}{N}\right) .
$$

(II)- The mapping $f \mapsto G_{\alpha} * f$ is continuous from $L^{p}\left(\mathbb{R}^{N}\right)$ to $L_{\sigma}^{q}\left(\mathbb{R}^{N}\right)$ if and only if

$$
\sigma(K)^{\frac{1}{q}} \leq c_{2}\left(c_{\alpha, p}(K)\right)^{\frac{1}{p}} \quad \text { for all } K \subset \mathbb{R}^{N},
$$

where $c_{\alpha, p}$ denotes the Bessel capacity of order $\alpha$ defined in (1.18). In fact this holds if and only if

$$
\sigma\left(B_{r}(x)\right) \leq c_{3}\left(c_{\alpha, p}\left(B_{r}(x)\right)\right)^{q / p} \quad \text { for all } x \in \mathbb{R}^{N}, 0<r \leq 1 .
$$

(III)- A necessary and sufficient condition in order the mapping $f \mapsto G_{\alpha} * f$ be compact from $L^{p}\left(\mathbb{R}^{N}\right)$ to $L_{\sigma}^{q}\left(\mathbb{R}^{N}\right)$ is

$$
\begin{aligned}
& \lim _{\delta \rightarrow 0} \sup _{x \in \mathbb{R}^{N}, r \leq \delta} \frac{\sigma\left(B_{r}(x)\right)}{\left(c_{\alpha, p}\left(B_{r}(x)\right)\right)^{\frac{q}{p}}}=0 \\
& \lim _{|x| \rightarrow \infty} \sup _{r \leq 1} \frac{\sigma\left(B_{r}(x)\right)}{\left(c_{\alpha, p}\left(B_{r}(x)\right)\right)^{\frac{q}{p}}}=0 .
\end{aligned}
$$

If $\mathbb{R}^{N}$ is replaced by a smooth bounded set $\Omega$, we extend any bounded Radon measure in $\Omega$ by zero in $\Omega^{c}$. In view of $[2,5.6 .1]$ the $c_{I_{\alpha}, p^{p}}$-Riesz capacity and $c_{\alpha, p^{-}}$ Bessel capacity of balls $B_{r}(x)$ with $x \in \Omega$ and $r \leq 1$ are then equivalent. It follows that $c_{\alpha, p}\left(B_{r}(x)\right) \simeq r^{N-\alpha p}$. Then, it follows from II and III above, the definition of $H^{\alpha, p}\left(\mathbb{R}^{N}\right)$ and the existence of an extension operator $H^{\alpha, p}(\Omega) \hookrightarrow H^{\alpha, p}\left(\mathbb{R}^{N}\right)$ that the following holds, 
Proposition 2.4 Under the assumptions of Proposition 2.3, the embedding $H^{\alpha, p}(\Omega) \hookrightarrow$ $L_{\sigma}^{q}(\Omega)$ is:

(I)- continuous if and only if $(\sigma(K))^{\frac{1}{q}} \leq c_{2}\left(c_{\alpha, p}(K)\right)^{\frac{1}{p}}$ for all $K \subset \mathbb{R}^{N}$, i.e. if and only if $\sigma \in \mathcal{M}_{r}^{+}\left(\mathbb{R}^{N}\right)$ with $\frac{1}{r}=q\left(\frac{1}{q}-\frac{1}{p}+\frac{\alpha}{N}\right)$.

(II)- compact if and only if

$$
\limsup _{r \rightarrow 0} \frac{\sigma\left(B_{r}(x)\right)}{r^{\frac{(N-\alpha p) q}{p}}}=0 .
$$

As an immediate corollary,

Proposition 2.5 Let $\sigma \in \mathcal{M}_{\frac{N}{N-\theta}}^{+}(\Omega)$, i.e. $\sigma\left(B_{r}(x)\right) \leq c r^{\theta}, N>\alpha p$ and $1<p<$ $q<\frac{N p}{N-\alpha p}$. Then the embedding

$$
H^{\alpha, p}(\Omega) \hookrightarrow L_{\sigma}^{q}(\Omega),
$$

is continuous iff $\sigma(K) \leq c_{1}\left(c_{\alpha, p}(K)\right)^{\frac{q}{p}}$ for all $K \subset \mathbb{R}^{N}$ which holds iff $q \leq \frac{\theta p}{N-\alpha p}$. And the embedding (2.16) is compact iff $q<\frac{\theta p}{N-\alpha p}$.

Other trace inequalities can be found in [21]. In the case $N=\alpha p$ the following estimate holds, see e.g. [1], [20, Corollary 8.6.2], [31].

Proposition 2.6 Let $\sigma$ be a nonnegative Radon measure in $\mathbb{R}^{N}$ with compact support and $N=\alpha p, p>1$. Then there exists a constant $b=b(N, \alpha, p)>0$ such that

$$
\sup _{\|f\|_{L^{p} \leq 1}} \int_{\mathbb{R}^{N}} \exp \left(b\left|G_{\alpha} * f\right|^{p^{\prime}}\right) d \sigma<\infty
$$

if and only if $\sigma \in \mathcal{M}_{\tau}^{+}\left(\mathbb{R}^{N}\right)$ for some $\tau \in(1, \infty)$.

When $p=1$ the next result is proved in [20, Sec 1.4.3]

Proposition 2.7 Let $\sigma$ be a nonnegative bounded Radon measure in $\mathbb{R}^{N}$, $\alpha$ be an integer such that $1 \leq \alpha \leq N$ and $q \geq 1$. Then the following estimate holds

$$
\|f\|_{L_{\sigma}^{q}} \leq c_{2} \sum_{|\beta|=\alpha}\left\|D^{\alpha} f\right\|_{1} \quad \text { for all } f \in C_{0}^{\infty}\left(\mathbb{R}^{N}\right)
$$

for some $c_{2}=c_{2}(N, p, q, \alpha)>0$ if and only if $\sigma \in \mathcal{M}_{\overline{N-q(N-\alpha)}}^{+}\left(\mathbb{R}^{N}\right)$. 


\section{The subcritical case}

\subsection{The variational construction}

We prove in this section that if $\mu \in W^{-1,2}(\Omega)$ then, under some assumptions on $g$ and $\sigma$, equation (1.2) has a variational solution.

We assume that $g \in C(\mathbb{R})$ satisfies $(1.1)$, and set $G(r):=\int_{0}^{r} g(s) d s$. We will find a solution to (1.2) minimizing the functional

$$
J(v):=\frac{1}{2} \int_{\Omega}|\nabla v|^{2} d x+\int_{\Omega} G(v) d \sigma-\langle\mu, v\rangle,
$$

over the set

$$
X_{G}(\Omega):=\left\{v \in W_{0}^{1,2}(\Omega): G(v) \in L_{\sigma}^{1}(\Omega)\right\} .
$$

The next proposition is a variant of a result in [8].

Proposition 3.1 Assume $\sigma \in \mathcal{M}_{\frac{N}{N-\theta}}^{+}(\Omega)$ with $N \geq \theta>\frac{N}{2}-1$. If $\mu \in W^{-1,2}(\Omega)$ there exists $u \in X_{G}(\Omega)$ which minimizes $J$ in $X_{G}(\Omega)$. Furthermore $u$ is a weak solution of (1.2) in the sense that

$$
\int_{\Omega} \nabla u . \nabla \zeta d x+\int_{\Omega} g(u) \zeta d \sigma=\langle\mu, \zeta\rangle \quad \text { for all } \zeta \in C_{0}^{\infty}(\Omega) .
$$

If $g$ is nondecreasing this solution is unique and denoted by $u_{\mu}$, and the mapping $\mu \mapsto u_{\mu}$ is nonnecreasing.

Proof. Step 1: Existence of a minimizer. If $N>2$ we apply (2.16) with $\alpha=1$ and $p=2$, recalling that by Fourier transform $H^{1,2}(\Omega)=W^{1,2}(\Omega)$ (it is a special case of Calderón's theorem), to obtain that

$$
W_{0}^{1,2}(\Omega) \hookrightarrow L_{\sigma}^{\frac{2 \theta}{N-2}}(\Omega) .
$$

If $N=2$ with $p=2$ we take any $\alpha<1$ and obtain

$$
\|f\|_{L_{\sigma}^{\frac{\theta}{1-\alpha}}} \leq c_{1}\|f\|_{W^{\alpha, 2}} \leq c_{1}^{\prime}\|f\|_{W^{1,2}} .
$$

According to Proposition 2.5 the imbedding of $W_{0}^{1,2}(\Omega)$ into $L_{\sigma}^{p}(\Omega)$ is compact for any $p \in\left[1, \frac{2 \theta}{N-2}\right)$ if $N>2$ and $1 \leq p<\infty$ if $N=2$.

Let us first assume that $g$ is bounded. Then $|G(v)| \leq m|v|$. Since $g$ is continuous, $G(v) \in L_{\sigma}^{1}(\Omega)$ for any $v \in W_{0}^{1,2}(\Omega)$ and the functional $J$ is well defined and is of class $C^{1}$ in $W_{0}^{1,2}(\Omega)$. Furthermore

$$
\lim _{\|v\|_{W^{1,2} \rightarrow \infty}} J(v)=+\infty .
$$


Let $\left\{u_{n}\right\}$ be a minimizing sequence. By (3.6), $\left\{u_{n}\right\}$ is bounded in $W_{0}^{1,2}(\Omega)$ and thus relatively compact in $L_{\sigma}^{1}(\Omega)$ and in $L^{2}(\Omega)$. Hence there exist $u \in L^{2}(\Omega)$ and $v \in L_{\sigma}^{1}(\Omega)$ such that, up to a subsequence, $u_{n} \rightarrow v$ in $L_{\sigma}^{1}(\Omega)$, and $u_{n} \rightarrow u$ strongly in $L^{2}(\Omega)$ and weakly in $W_{0}^{1,2}(\Omega)$. We can also assume that $u_{n} \rightarrow u c_{1,2^{2}}$ quasi almost everywhere in the sense that there exists $E \subset \Omega$ with $c_{1,2}(E)=0$ such that $u_{n}(x) \rightarrow u(x)$ for any $x \in \Omega \backslash E$. According to Proposition 2.5, $\sigma$ is absolutely continuous with respect to the $c_{1,2}$-capacity. It follows that $\sigma(E)=0$ so that $u_{n} \rightarrow u$ $\sigma$-almost everywhere and thus $u=v \sigma$-almost everywhere. Thus we have that $u_{n} \rightarrow u$ in $L^{2}(\Omega)$, in $L_{\sigma}^{1}(\Omega), \sigma$-almost everywhere and weakly in $W_{0}^{1,2}(\Omega)$. Then we have that $\left\langle\mu, u_{n}\right\rangle \rightarrow\langle\mu, u\rangle$. By the dominated convergence theorem we have also that $G\left(u_{n}\right) \rightarrow G(u)$ in $L_{\sigma}^{1}(\Omega)$. Therefore

$$
J(u) \leq \liminf _{n \rightarrow \infty} J\left(u_{n}\right),
$$

which implies that $u$ is a minimizer of $J$ in $W_{0}^{1,2}(\Omega)$.

If $g$ is unbounded, we write $g=g_{1}+g_{2}$ where $g_{1}=g \chi_{\left(-r_{0}, r_{0}\right)}, g_{2}=g \chi_{\left(-\infty-r_{0}\right] \cup\left[r_{0}, \infty\right)}$, where $r_{0}$ is defined in (1.1). Hence $G(r)=G_{1}(r)+G_{2}(r)$ where $\left|G_{1}(r)\right| \leq m|r|$ and $G_{2}(r)$ is nonnegative. Using again (2.14) we obtain that (3.6) holds. A minimizing sequence $\left\{u_{n}\right\}$ inherits the same property as above, hence $u_{n} \rightarrow u \sigma$-almost everywhere in $\Omega$ and in $L_{\sigma}^{1}(\Omega)$, this implies that $G_{1}\left(u_{n}\right) \rightarrow G_{1}(u)$ in $L_{\sigma}^{1}(\Omega)$ and $G_{2}(u)$ is $\sigma$-measurable. By Fatou's lemma

$$
\int G_{2}(u) d \sigma \leq \liminf _{n \rightarrow \infty} \int G_{2}\left(u_{n}\right) d \sigma
$$

which implies that (3.7) holds. Notice that, among the consequences, $X_{G}$ is closed subset of $W_{0}^{1,2}(\Omega)$. Hence $u$ in a minimizer of $J$ in $X_{G}(\Omega)$.

Uniqueness holds if $g$ is nondecreasing since it implies that $J$ is stricly convex and actually $X_{G}$ is a closed convex set.

Step 2: The minimizer is a weak solution. For $k>r_{0}$ we define $g_{k}$ by

$$
g_{k}(r)= \begin{cases}g(r) & \text { if }|r| \leq k \\ g(k) & \text { if } r>k \\ g(-k) & \text { if } r<-k\end{cases}
$$

Then $g_{k}$ is continuous and bounded and the minimizer $u_{k} \in W_{0}^{1,2}(\Omega)$ of

$$
J_{k}(v)=\frac{1}{2} \int_{\Omega}|\nabla v|^{2} d x+\int_{\Omega} G_{k}(v) d \sigma-\langle\mu, v\rangle \text { where } G_{k}(r)=\int_{0}^{s} g_{k}(s) d s,
$$

is a weak solution (i.e. in the sense given by (3.3)) of

$$
\begin{aligned}
-\Delta u+g_{k}(u) \sigma & =\mu & & \text { in } \Omega \\
u & =0 & & \text { on } \partial \Omega .
\end{aligned}
$$


The following energy estimate holds

$$
\int_{\Omega}\left|\nabla u_{k}\right|^{2} d x+\int_{\Omega} u_{k} g_{k}\left(u_{k}\right) d \sigma=\left\langle\mu, u_{k}\right\rangle \leq\|\mu\|_{W^{-1,2}}\left\|u_{k}\right\|_{W^{1,2}}
$$

and it implies

$$
\int_{\Omega}\left|\nabla u_{k}\right|^{2} d x+\int_{\Omega}\left|u_{k} g_{k}\left(u_{k}\right)\right| d \sigma \leq\|\mu\|_{W^{-1,2}}^{2}+m \sigma(\Omega)=M
$$

for some $m=m\left(r_{0}\right)>0$. Up to a subsequence, $\left\{u_{k}\right\}_{k}$ converges to some $u$ as $k \rightarrow \infty$, weakly in $W_{0}^{1,2}(\Omega)$, strongly in $L^{2}(\Omega)$, and almost everywhere in $\Omega$. By Proposition 2.4 the imbedding of $W^{1,2}(\Omega)$ in $L_{\sigma}^{q}(\Omega)$ is compact for any $q<\frac{2 \theta}{N-2}$. Hence the subsequence can be taken such that $u_{k} \rightarrow u, \sigma$-almost everywhere as $k \rightarrow \infty$, and consequently $g_{k}\left(u_{k}\right) \rightarrow g(u) \sigma$-almost everywhere. Let $E \subset \Omega$ be a Borel set, then for any $\lambda>r_{0}$,

$$
\begin{aligned}
M & \geq \int_{E}\left|g_{k}\left(u_{k}\right) u_{k}\right| d \sigma \\
& =\int_{E \cap\left\{\left|u_{k}\right|>\lambda\right\}}\left|g_{k}\left(u_{k}\right) u_{k}\right| d \sigma+\int_{E \cap\left\{\left|u_{k}\right| \leq \lambda\right\}}\left|g_{k}\left(u_{k}\right) u_{k}\right| d \sigma \\
& \geq \lambda \int_{E \cap\left\{\left|u_{k}\right|>\lambda\right\}}\left|g_{k}\left(u_{k}\right)\right| d \sigma+\int_{E \cap\left\{\left|u_{k}\right| \leq \lambda\right\}}\left|g_{k}\left(u_{k}\right) u_{k}\right| d \sigma .
\end{aligned}
$$

Therefore

$$
\begin{aligned}
\int_{E}\left|g_{k}\left(u_{k}\right)\right| d \sigma & =\int_{E \cap\left\{\left|u_{k}\right|>\lambda\right\}}\left|g_{k}\left(u_{k}\right)\right| d \sigma+\int_{E \cap\left\{\left|u_{k}\right| \leq \lambda\right\}}\left|g_{k}\left(u_{k}\right)\right| d \sigma \\
& \leq \frac{M}{\lambda}+\max \{|g(r)|:|r| \leq \lambda\} \sigma(E)
\end{aligned}
$$

For $\epsilon>0$ we first choose $\lambda$ such that $\frac{M}{\lambda} \leq \frac{\epsilon}{2}$ and then $\sigma(E) \leq \frac{\epsilon}{1+2 \max \{|g(r)| \leq \lambda\}}$. This implies the uniform integrability of $\left\{g_{k}\left(u_{k}\right)\right\}_{k}$ in $L_{\sigma}^{1}(\Omega)$. Hence $g_{k}\left(u_{k}\right) \rightarrow g(u)$ in $L_{\sigma}^{1}(\Omega)$ by Vitali's convergence theorem. Since $u_{k}$ is a weak solution of $(3.8)$, there holds for any $\zeta \in C_{0}^{\infty}(\Omega)$,

$$
\int_{\Omega} \nabla u_{k} \cdot \nabla \zeta d x+\int_{\Omega} g_{k}\left(u_{k}\right) \zeta d \sigma=\langle\mu, \zeta\rangle .
$$

Letting $k \rightarrow \infty$ we obtain, using the above convergence results,

$$
-\int_{\Omega} \nabla u . \nabla \zeta d x+\int_{\Omega} g(u) \zeta d \sigma=\langle\mu, \zeta\rangle .
$$

Hence $u$ is a weak solution. If $g$ is monotone, uniqueness is also a consequence of the weak formulation. Furthermore if $\mu, \mu^{\prime}$ belong to $W^{-1,2}(\Omega)$ are such that $\mu-\mu^{\prime}$ is a nonnegative measure, then $\left\langle\mu^{\prime}-\mu,\left(u_{\mu}^{\prime}-u_{\mu}\right)_{+}\right\rangle \leq 0$. Taking $\left(u_{\mu}^{\prime}-u_{\mu}\right)_{+}$for test function in the weak formulation yields $\left(u_{\mu}^{\prime}-u_{\mu}\right)_{+}=0$. 


\subsection{The $L^{1}$ case}

In the sequel we set

$$
\mathbb{X}(\Omega)=\left\{\zeta \in C^{1}(\bar{\Omega}), \zeta=0 \text { on } \partial \Omega \text { and } \Delta \zeta \in L^{\infty}(\Omega)\right\},
$$

and $\mathbb{X}_{+}(\Omega)=\mathbb{X}(\Omega) \cap\left\{\zeta \in C^{1}(\bar{\Omega}): \zeta \geq 0\right.$ in $\left.\bar{\Omega}\right\}$. We recall (see e.g. [29]) that if $f \in L_{\rho}^{1}(\Omega)$ and $u \in L^{1}(\Omega)$ is a very weak solution of

$$
-\Delta u=f \quad \text { in } \Omega,
$$

there holds

$$
-\int_{\Omega}|u| \Delta \zeta d x \leq \int_{\Omega} f \operatorname{sign}(u) \zeta d x \quad \text { for all } \zeta \in \mathbb{X}_{+}(\Omega)
$$

and

$$
-\int_{\Omega} u^{+} \Delta \zeta d x \leq \int_{\Omega} f \operatorname{sign}_{+}(u) \zeta d x \quad \text { for all } \zeta \in \mathbb{X}_{+}(\Omega)
$$

Proposition 3.2 Assume $N \geq 2, \sigma \in \mathcal{M}_{\frac{N}{N-\theta}}^{+}(\Omega)$ with $N \geq \theta>N-2$ and $g: \mathbb{R} \mapsto$ $\mathbb{R}$ is a continuous nondecreasing function vanishing at 0 . If $\mu \in L_{\rho}^{1}(\Omega)$ there exists a unique $u:=u_{\mu} \in L^{1}(\Omega)$ very weak solution of (1.2). Furthermore, if $u_{\mu}, u_{\mu^{\prime}} \in L^{1}(\Omega)$ are the very weak solutions of (1.2) with right-hand sides $\mu, \mu^{\prime} \in L_{\rho}^{1}(\Omega)$, then

$$
-\int_{\Omega}\left|u_{\mu}-u_{\mu^{\prime}}\right| \Delta \zeta d x+\int_{\Omega}\left|g\left(u_{\mu}\right)-g\left(u_{\mu^{\prime}}\right)\right| \zeta d \sigma \leq \int_{\Omega}\left(\mu-\mu^{\prime}\right) \operatorname{sign}\left(u_{\mu}-u_{\mu^{\prime}}\right) \zeta d x
$$

and

$$
-\int_{\Omega}\left(u_{\mu}-u_{\mu^{\prime}}\right)_{+} \Delta \zeta d x+\int_{\Omega}\left(g\left(u_{\mu}\right)-g\left(u_{\mu^{\prime}}\right)\right)_{+} \zeta d \sigma \leq \int_{\Omega}\left(\mu-\mu^{\prime}\right) \operatorname{sign}_{+}\left(u_{\mu}-u_{\mu^{\prime}}\right) \zeta d x
$$

for any $\zeta \in \mathbb{X}_{+}(\Omega)$. In particular the mapping $\mu \rightarrow u_{\mu}$ is nondecreasing.

The following result will be used several time in the sequel. Its proof is standard but we present it for the sake of completeness.

Lemma 3.3 Assume $N>q \geq 1$ and $\sigma \in \mathcal{M}_{\frac{N}{N-\theta}}^{+}$with $N \geq \theta>N-q$. Then $\sigma$ vanishes on any Borel set with $c_{1, q}$-capacity zero.

Proof. It suffices to prove the result when $E$ is compact. We define the $\Lambda_{\theta}$ Hausdorff measure of a set $E$ by

$$
\Lambda_{\theta}(E)=\lim _{\kappa \rightarrow 0} \Lambda_{\theta}^{\kappa}(E):=\lim _{\kappa \rightarrow 0} \inf \left\{\sum_{j=1}^{\infty} r_{j}^{\theta}: 0<r_{j} \leq \kappa \leq \infty, E \subset \bigcup_{j=1}^{\infty} B_{r_{j}}\left(a_{j}\right)\right\} .
$$


Note that $\Lambda_{\theta}^{\infty}(E)$ is the Hausdorff content of $E$ and it is smaller than $(\operatorname{diam}(E))^{\theta}$. For any covering of $E$ by balls $B_{r_{j}}\left(a_{j}\right), j \geq 1$, we have

$$
\sigma(E) \leq \sum_{j=1}^{\infty} \sigma\left(B_{r_{j}}\left(a_{j}\right)\right) \leq\|\sigma\|_{\frac{N}{N-\theta}} \sum_{j=1}^{\infty} r_{j}^{\theta} .
$$

It follows that

$$
\sigma(E) \leq\|\sigma\|_{\frac{N}{N-\theta}} \Lambda_{\theta}(E) .
$$

Next, if $c_{1, q}(E)=0$ then $\Lambda_{\theta}(E)=0$ according to [2, Th. 5.1.13], and thus $\sigma(E)=0$ by the previous inequality.

We introduce the flow coordinates near $\partial \Omega$ defined by

$$
\Pi(x)=(\rho(x), \tau(x)) \in\left[0, \epsilon_{0}\right] \times \partial \Omega \quad \text { where } \tau(x)=\operatorname{proj}_{\partial \Omega}(x) .
$$

It is well-known that for $\epsilon_{0}$ small enough, $\Pi$ is a $C^{1}$-diffeomorphism from $\Omega_{\epsilon_{0}}:=$ $\left\{x \in \bar{\Omega}: \rho(x) \leq \epsilon_{0}\right\}$ to $\left[0, \epsilon_{0}\right] \times \partial \Omega$. With this diffeomorphism we can assimilate the surface measure $d S_{\epsilon}$ on $\Sigma_{\epsilon}=\{x \in \Omega: \rho(x)=\epsilon\}$ with the surface measure $d S$ on $\Sigma_{0}=\partial \Omega$ by setting

$$
\int_{\Sigma_{\epsilon}} v(x) d S_{\epsilon}(x)=\int_{\Sigma_{0}} v(\epsilon, \tau) d S(\tau)
$$

Lemma 3.4 Assume $N \geq 2$ and $\mu \in \mathfrak{M}(\Omega)$ satisfies

$$
\int_{\Omega} \rho d|\mu|<\infty
$$

Then $u=\mathbb{G}[\mu]$ satisfies

$$
\lim _{\epsilon \rightarrow 0} \int_{\Sigma_{0}}|u|(\epsilon, \tau) d S(\tau)=0
$$

Proof. If $u=\mathbb{G}[\mu]$, it is the unique weak solution of $-\Delta u=\mu$ in $\Omega, u=0$ on $\partial \Omega$. Hence $u=u_{1}-u_{2}$ where $u_{1}=\mathbb{G}\left[\mu^{+}\right]$and $u_{2}=\mathbb{G}\left[\mu^{-}\right]$. Since $\mu_{+}$and $\mu_{-}$satisfy the integrability condition (3.20) both $u_{1}$ and $u_{2}$ have a zero measure boundary trace ( $M$-boundary trace in the sense of $[18$, Sec 1.3$])$. Hence, taking for test function the function $\zeta=1$,

$$
\lim _{\epsilon \rightarrow 0} \int_{\Sigma_{0}} u_{j}(\epsilon, \tau) d S(\tau)=0
$$

which implies (3.20).

This result allows us to obtain the uniqueness of the solution even if the righthand side is a measure. 
Lemma 3.5 Assume $N \geq 2, \sigma \in \mathcal{M}_{\frac{N}{N-\theta}}^{+}(\Omega)$ with $N \geq \theta>N-2$ and $g: \mathbb{R} \mapsto \mathbb{R}$ is a continuous nondecreasing function. If $\mu \in \mathfrak{M}(\Omega)$ there exists at most one very weak solution of (1.2).

Proof. By Lemma 3.3 with $\alpha=1, p=2, \sigma$ is absolutely continuous with respect to the $c_{1,2}$ capacity (it is diffuse in the terminology of [9]), and if $h \in L_{\sigma}^{1}(\Omega)$ the measure $h_{+} \sigma$, which is the increasing limit of $\inf \left\{n, h_{+}\right\} \sigma$ is also diffuse. Similarly $h_{-} \sigma$ is diffuse and so is $h \sigma$. Next we assume that $u$ and $u^{\prime}$ are two very weak solutions of (1.2) and set $w=u-u^{\prime}$. Hence

$$
-\Delta w+\left(g(u)-g\left(u^{\prime}\right)\right) \sigma=0 .
$$

Since $\rho\left(g(u)-g\left(u^{\prime}\right)\right) \in L_{\sigma}^{1}(\Omega)$, it follows from Lemma 3.4 that

$$
\lim _{\epsilon \rightarrow 0} \int_{\Sigma_{\epsilon}}|w|(\epsilon, \tau) d S(\tau)=0
$$

We use Kato inequality for measures as in [10, Th 1.1]: Since $w \in L^{1}(\Omega), \Delta w^{+}$is a diffuse measure and

$$
\Delta w^{+} \geq \chi_{\{w \geq 0\}} \Delta w=\chi_{\{w \geq 0\}}\left(g(u)-g\left(u^{\prime}\right)\right) \sigma \geq 0 \text { in } \Omega
$$

Since $w^{+}$has a M-boundary trace by Lemma 3.4, we can apply [18, Lemmma 1.5.8] with $\mu=-\chi_{\{w \geq 0\}}\left(g(u)-g\left(u^{\prime}\right)\right) \sigma$ which is a measure in $\mathfrak{M}_{\rho}(\Omega):=\{\nu \in \mathfrak{M}(\Omega): \rho \nu \in$ $\left.\mathfrak{M}_{b}(\Omega)\right\}$. Then there exists $\tau \in \mathfrak{M}_{\rho}^{+}(\Omega)$ such that

$$
-\Delta w^{+}=\mu-\tau \text {. }
$$

Equivalently

$$
-\Delta w^{+}+\chi_{\{w \geq 0\}}\left(g(u)-g\left(u^{\prime}\right)\right) \sigma=-\tau .
$$

Since the M-boundary trace of $w^{+}$is zero, it follows that $w^{+}=-\mathbb{G}\left[\chi_{\{w \geq 0\}}(g(u)-\right.$ $\left.g\left(u^{\prime}\right)\right) \sigma+\tau$. Hence $w^{+}=0$ and $u \leq u^{\prime}$. Similarly $u^{\prime} \leq u$.

The following variant will be useful in the sequel.

Lemma 3.6 Assume $N \geq 2, \sigma \in \mathcal{M}_{\frac{N}{N-\theta}}^{+}(\Omega)$ with $N \geq \theta>N-2$ and $g: \mathbb{R} \mapsto \mathbb{R}$ is a continuous nondecreasing function. If $u, u^{\prime} \in L^{1}(\Omega)$ are such that $\rho g(u)$ and $\rho g\left(u^{\prime}\right)$ belong to $L_{\sigma}^{1}(\Omega)$ and satisfy

$$
-\int_{\Omega}\left(u-u^{\prime}\right) \Delta \zeta d x+\int_{\Omega}\left(g(u)-g\left(u^{\prime}\right)\right) \zeta d \sigma=\int_{\Omega} \zeta d \nu \quad \text { for all } \zeta \in \mathbb{X}_{+}(\Omega)
$$

for some $\nu \in \mathfrak{M}_{+}(\Omega)$ diffuse with respect to the $c_{1,2}$-capacity, then $u \geq u^{\prime} c_{1,2}$-quasi everywhere in $\Omega$. 
Proof. We use Kato's inequality, Lemma 3.4 and [18, Lemma 1.5.8] in the same way as in the proof of Lemma 3.5 since the measures $\left(g(u)-g\left(u^{\prime}\right)\right) d \sigma$ and $\nu$ are diffuse, $\Delta\left(u^{\prime}-u\right)$ is diffuse, hence

$$
\Delta\left(u^{\prime}-u\right)_{+} \geq \chi_{\left\{u^{\prime} \geq u\right\}} \Delta\left(u^{\prime}-u\right)=\left(g\left(^{\prime}\right)-g(u)\right) \chi_{\left\{u^{\prime} \geq u\right\}}+\chi_{\left\{u^{\prime} \geq u\right\}} \nu \geq 0
$$

Since $u^{\prime}-u \in W_{0}^{1, q}(\Omega)$ for any $1<q<\frac{N}{N-1}$, we conclude that $\left(u^{\prime}-u\right)_{+}=0$ almost everywhere and $c_{1,2}$-quasi everywhere by [2, Th 6.1.4].

The next result and the corollary which follows are the key-stone for the proof of Proposition 3.2.

Lemma 3.7 Let $\sigma \in \mathcal{M}_{\frac{N}{N-\theta}}^{+}(\Omega)$ with $N \geq \theta>N-2, h \in L_{\sigma}^{\infty}(\Omega), f \in L^{s}(\Omega)$ with $s>\frac{N}{2}$ and $w \in L^{1}(\Omega)$ be the very weak solution of

$$
\begin{aligned}
-\Delta w+h \sigma=f & & \text { in } \Omega \\
w=0 & & \text { in } \partial \Omega .
\end{aligned}
$$

Then $w$ is continuous in $\bar{\Omega}$ and for any nondecreasing bounded function $\gamma \in C^{2}(\mathbb{R})$ vanishing at 0 , there holds

$$
-\int_{\Omega} j(w) \Delta \zeta d x+\int_{\Omega} \gamma(w) h \zeta d \sigma \leq \int_{\Omega} \gamma(w) \zeta f d x \quad \text { for all } \zeta \in \mathbb{X}_{+}(\Omega)
$$

where $j(r)=\int_{0}^{r} \gamma(s) d s$.

Proof. The solution is unique and expressed by $w=\mathbb{G}[f-h \sigma]$. Since $\frac{N}{N-\theta}>\frac{N}{2}$, $w \in C^{\alpha}(\bar{\Omega})$ for some $\alpha \in(0,1)$ by Lemma 2.2. Hence $\gamma(w)$ is continuous and therefore measurable. We extend $\sigma$ by zero in $\Omega^{c}$ and denote $\sigma_{n}=\sigma * \eta_{n}$ where $\left\{\eta_{n}\right\}$ is a sequence of mollifiers. Then $\sigma_{n} \rightarrow \sigma$ in the narrow topology of $\Omega$. For $n \in \mathbb{N}^{*}$, let $w_{n}$ be the solution of

$$
\begin{array}{cl}
-\Delta w_{n}+h \sigma_{n}=T_{n}(f) & \text { in } \Omega \\
w_{n}=0 & \text { in } \partial \Omega,
\end{array}
$$

where $T_{n}(f)=\min \{|f|, n\} \operatorname{sgn}(f)$. Then $w_{n} \in W^{2, s}(\Omega) \cap W_{0}^{1, \infty}(\Omega)$ for all $1<s<\infty$. By Green's formula

$$
-\int_{\Omega} j\left(w_{n}\right) \Delta \zeta d x+\int_{\Omega} \gamma\left(w_{n}\right) h \zeta d \sigma \leq \int_{\Omega} \gamma\left(w_{n}\right) \zeta f d x \quad \text { for all } \zeta \in \mathbb{X}_{+}(\Omega)
$$

Since $w_{n} \rightarrow w$ uniformly in $\bar{\Omega},(3.25)$ follows. 
Corollary 3.8 Under the assumptions of Lemma 3.7, there holds

$$
-\int_{\Omega}|w| \Delta \zeta d x+\int_{\Omega} \operatorname{sign}_{0}(w) h \zeta d \sigma \leq \int_{\Omega} \operatorname{sign}_{0}(w) \zeta f d x
$$

and

$$
-\int_{\Omega} w_{+} \Delta \zeta d x+\int_{\Omega} \operatorname{sign}_{+}(w) \zeta h d \sigma \leq \int_{\Omega} \operatorname{sign}_{+}(w) \zeta f d x,
$$

for any $\zeta \in \mathbb{X}_{+}(\Omega)$. Moreover there exists a constant $C>0$ depending only on $\Omega$ such that

$$
\int_{\Omega} \operatorname{sign}_{0}(w) h d \sigma \leq C \int_{\Omega}|f| d x
$$

Proof. For proving (3.28) we consider a sequence $\left\{\gamma_{k}\right\}$ of odd nondecreasing functions such that

$$
\gamma_{k}(r)=\left\{\begin{aligned}
1 & \text { if } r \geq 2 k^{-1} \\
0 & \text { if }-k^{-1} \leq r \leq k^{-1} \\
-1 & \text { if } r \leq-2 k^{-1}
\end{aligned}\right.
$$

and such that $\left\{r \gamma_{k}(r)\right\}$ is nondecreasing for any $r$. Using $\gamma_{k}$ in place of $\gamma$ in (3.25) we obtain

$$
-\int_{\Omega} j_{k}(w) \Delta \zeta d x+\int_{\Omega} \gamma_{k}(w) \zeta h d \sigma \leq \int_{\Omega} \gamma_{k}(w) \zeta f d x \quad \text { for all } \zeta \in \mathbb{X}_{+}(\Omega)
$$

where $j_{k}(r)=\int_{0}^{r} \gamma_{k}(s) d s$. Since $\gamma_{k}(w) \uparrow w$ on $\Omega_{+}:=\{x \in \Omega: w(x)>0\}$, there holds by the monotone convergence theorem,

$$
\int_{\Omega_{+}} \gamma_{k}(w) \zeta|h| d \sigma \uparrow \int_{\Omega_{+}} w \zeta|h| d \sigma \quad \text { as } k \rightarrow \infty .
$$

Since

$$
\left|\int_{\Omega_{+}}\left(w-\gamma_{k}(w)\right) \zeta h d \sigma\right| \leq \int_{\Omega_{+}}\left|\left(w-\gamma_{k}(w)\right) \zeta h\right| d \sigma=\int_{\Omega_{+}}\left(w-\gamma_{k}(w)\right) \zeta|h| d \sigma,
$$

we obtain

$$
\int_{\Omega_{+}} \gamma_{k}(w) h \zeta d \sigma \rightarrow \int_{\Omega_{+}} w h \zeta d \sigma \quad \text { as } k \rightarrow \infty
$$

Similarly, $\gamma_{k}(w) \downarrow w$ on $\Omega_{-}:=\{x \in \Omega: w(x)<0\}$ so that

$$
\int_{\Omega_{-}} \gamma_{k}(w) h \zeta d \sigma \rightarrow \int_{\Omega_{-}} w h \zeta d \sigma \quad \text { as } k \rightarrow \infty
$$


Combining these two results yields

$$
\int_{\Omega} \gamma_{k}(w) \zeta h d \sigma \rightarrow \int_{\Omega_{+}} w \zeta h d \sigma-\int_{\Omega_{-}} w \zeta h d \sigma=\int_{\Omega} \operatorname{sign}_{0}(w) \zeta h d \sigma .
$$

Usiing dominated convergence theorem there holds

$$
\int_{\Omega} \gamma_{k}(w) \Delta \zeta d x \rightarrow \int_{\Omega} \operatorname{sign}_{0}(w) \Delta \zeta d x
$$

and

$$
\int_{\Omega} \gamma_{k}(w) \zeta f d x \rightarrow \int_{\Omega} \operatorname{sign}_{0}(w) \zeta f d x
$$

This implies (3.28). The proof of (3.17) is similar.

Eventually we prove (3.30). Let $\eta_{1}$ be the solution of

$$
\begin{aligned}
& -\Delta \eta_{1}=1 \quad \text { in } \Omega \\
& \eta_{1}=0 \quad \text { in } \partial \Omega \text {. }
\end{aligned}
$$

Then $\eta_{1}=\mathbb{G}[1] \in \mathbb{X}_{+}(\Omega)$ and there exists $c, c^{\prime}>0$ depending only on $\Omega$ such that $c \rho \leq \eta_{1} \leq c^{\prime} \rho$. Given $\alpha \in(0,1]$, let $j_{\epsilon}(r)=(r+\epsilon)^{\alpha}-\epsilon^{\alpha}, r \geq 0$, and $\zeta=j_{\epsilon}\left(\eta_{1}\right)$. Note that $\zeta \in C^{2}(\bar{\Omega}), 0 \leq \zeta \leq \eta^{\alpha}, \zeta=0$ on $\partial \Omega, j_{\epsilon}^{\prime}>0, j_{\epsilon}^{\prime \prime}<0$, so that $-\Delta \zeta=j_{\epsilon}^{\prime}\left(\eta_{1}\right)-j_{\epsilon}^{\prime \prime}\left(\eta_{1}\right)\left|\nabla \eta_{1}\right|^{2} \geq 0$. We deduce from (3.28) that

$$
\int_{\Omega} \operatorname{sign}_{0}(w)(\eta+\epsilon)^{\alpha} h d \sigma \leq \int_{\Omega} \operatorname{sign}_{0}(w) \eta^{\alpha}|f| d x+\epsilon^{\alpha} \int_{\Omega} \operatorname{sign}_{0}(w) h d \sigma .
$$

We obtain

$$
\int_{\Omega} \operatorname{sign}_{0}(w) \rho^{\alpha} h d \sigma \leq C \int_{\Omega} \rho^{\alpha}|f| d x+\epsilon^{\alpha}|\tilde{\sigma}(\Omega)|
$$

Letting $\epsilon \rightarrow 0$ and then $\alpha \rightarrow 0$ we infer the result by dominated convergence.

We are now in position to prove Proposition 3.2.

Proof of Proposition 3.2. We divide the proof into several steps.

Step 1: We assume that $\mu \in L^{\infty}(\Omega)$. Let $\left\{\eta_{n}\right\}$ be a sequence of molifiers and $\sigma_{n}=\sigma * \eta_{n}$. If $\mu \in L^{\infty}(\Omega)$, the solution $u_{n}=u_{n, \mu}$ of

$$
\begin{array}{rlrl}
-\Delta u_{n}+g\left(u_{n}\right) \sigma_{n} & =\mu & & \text { in } \Omega \\
u_{n}=0 & & \text { in } \partial \Omega,
\end{array}
$$

is continuous in $\bar{\Omega}$. Since

$$
-\mathbb{G}\left[\mu^{-}\right] \leq-u_{n}^{-} \leq 0 \leq u_{n}^{+} \leq \mathbb{G}\left[\mu^{+}\right]
$$


by the maximum principle, the sequence $\left\{u_{n}\right\}$ is uniformly bounded. Recalling that $g$ is nondecreasing we have that the sequence $\left\{g\left(u_{n}\right)\right\}$ is also uniformly bounded in $\Omega$, hence $g\left(u_{n}\right) \sigma_{n}$ is bounded in $\mathcal{M}_{\frac{N}{N-\theta}}(\Omega)$ independently of $n$, and from (2.9) it follows that $u_{n}$ is bounded in $C^{\alpha}(\bar{\Omega})$ for some $\alpha \in(0,1]$ independently of $n$. Up to some subsequence, $\left\{u_{n}\right\}$, and thus also $\left\{g\left(u_{n}\right)\right\}$, are then uniformly convergent in $\bar{\Omega}$ with limit $u=u_{\mu}$ and $g(u)=g\left(u_{\mu}\right)$. Because $\sigma * \eta_{n}$ converges to $\sigma$ in the narrow topology, $u_{\mu}$ is a very weak solution of (1.2). Notice that being continuous, $g(u)$ is measurable for the measure $\sigma$. By Lemma 3.5, $u_{\mu}$ is the unique solution of (1.2), hence the whole sequence $\left\{u_{\mu_{n}}\right\}$ converges to $u_{\mu}$. Applying Corollary 3.8 with $w=u, \tilde{\sigma}=\sigma$ and $\zeta=\eta_{1}$ yields

$$
\int_{\Omega}|u| d x+\int_{\Omega}|g(u)| \eta_{1} d \sigma \leq \int_{\Omega}|\mu| \eta_{1} d x
$$

and (3.29) with $\zeta=\eta_{1}$ gives

$$
\int_{\Omega}\left(u-u^{\prime}\right)_{+} d x+\int_{\Omega}\left(g(u)-g\left(u^{\prime}\right)\right)_{+} \eta_{1} d \sigma \leq \int_{\Omega} \eta_{1} \operatorname{sign}_{+}\left(u-u^{\prime}\right)\left(\mu-\mu^{\prime}\right)_{+} d x .
$$

which implies the monotonicity of the mapping $\mu \mapsto u_{\mu}$.

Step 2: We assume that $\mu \in L^{1}(\Omega)$ is bounded from below. Set $\ell=\operatorname{ess} \inf \mu$. For $k>0$ set $\mu_{k}=\min \{k, \mu\}$ and $u_{k}:=u_{\mu_{k}} \in L^{\infty}(\Omega)$. The sequence $\left\{\mu_{k}\right\}$ is nondecreasing, hence according to Step 1, the sequence $\left\{u_{k}\right\}$ is a nondecreasing sequence of continuous functions in $\bar{\Omega}$ bounded from below by $\ell \eta_{1}$, where $\eta_{1}$ is defined in (3.32). Its pointwise limit, denoted by $u$, is thus lower semicontinuous. Moreover $g\left(u_{k}\right) \rightarrow g(u)$ pointwise, hence $g(u)$ is lower semicontinuous and thus $\sigma$-measurable. Relation (3.35) applied to $\mu_{k}$ and $u_{k}$ gives

$$
\int_{\Omega}\left|u_{k}\right| d x+\int_{\Omega}\left|g\left(u_{k}\right)\right| \eta_{1} d \sigma \leq \int_{\Omega}\left|\mu_{k}\right| \eta_{1} d x
$$

Passing to the limit using Fatou's lemma in the left-hand side and the dominated convergence theorem in the right-hand side yields

$$
\int_{\Omega}|u| d x+\int_{\Omega}|g(u)| \eta_{1} d \sigma \leq \int_{\Omega}|\mu| \eta_{1} d x
$$

We deduce that $u \in L^{1}(\Omega)$ and $\rho g(u) \in L_{\sigma}^{1}(\Omega)$. We have indeed a more precise result. Since $g$ vanishes at $0 g\left(u_{k}\right)=g\left(u_{k}^{+}\right)+g\left(-u_{k}^{-}\right)$. Hence $\rho g\left(u_{k}^{+}\right) \rightarrow \rho g\left(u^{+}\right)$in $L_{\sigma}^{1}(\Omega)$ by the monotone convergence theorem. Furthermore $g\left(-u_{1}^{-}\right) \leq g\left(-u_{k}^{-}\right) \leq 0$, which implies that $\rho g\left(-u_{k}^{-}\right) \rightarrow \rho g\left(-u^{-}\right)$in $L_{\sigma}^{1}(\Omega)$ by the dominated convergence theorem which finally implies that $\rho g\left(u_{k}\right) \rightarrow \rho g(u)$ in $L_{\sigma}^{1}(\Omega)$. Using $\zeta \in \mathbb{X}_{+}(\Omega)$ as a 
test function in the very weak formulation of the equation satisfied by $u_{k}$ gives

$$
-\int_{\Omega} u_{k} \Delta \zeta d x+\int_{\Omega} g\left(u_{k}\right) \zeta d \sigma=\int_{\Omega} \zeta \mu_{k} d x
$$

Since $u_{k} \rightarrow u$ almost everywhere and $-l \eta_{1} \leq u_{k} \leq u$ with $u \in L^{1}(\Omega)$, we can pass to the limit in the first term to obtain $\int_{\Omega} u_{k} \Delta \zeta d x \rightarrow \int_{\Omega} u \Delta \zeta d x$. Because $\left|\mu_{k}\right| \leq|\mu| \in L^{1}(\Omega)$ and $\mu_{k} \rightarrow \mu$ almost everywhere, we can also pass to the limit in the last term: $\int_{\Omega} \zeta \mu_{k} d x \rightarrow \int_{\Omega} \zeta \mu d x$. It remains to pass to the limit in the nonlinearity. Because $u_{k} \uparrow u$ and $g$ is nondecreasing, we have $g\left(u_{k}\right) \uparrow g(u)$. Thus by the monotone convergence theorem,

$$
-\int_{\Omega} u \Delta \zeta d x+\int_{\Omega} g(u) \zeta d \sigma=\int_{\Omega} \zeta \mu d x
$$

and $u$ is very weak solution of (1.2).

Step 3: We assume that $\mu \in L^{1}(\Omega)$. For $\ell \in \mathbb{R}$, we set $\mu^{\ell}=\sup \{\mu, \ell\}$ and denote by $u^{\ell}$ the solution of (1.2) with right-hand side $\mu^{\ell}$. Note that the sequence $\left\{\mu^{\ell}\right\}_{\ell}$ is increasing, bounded from above by $\mu^{+}$so that $u^{\ell} \leq u_{\mu^{+}}$, where $u_{\mu^{+}}$is the solution of (1.2) with right-hand side $\mu^{+}$which exists according to the previous step, and the sequence $\left\{u^{\ell}\right\}_{\ell}$ is monotone nondecreasing with $\ell$ with pointwise limit $u$ when $\ell \rightarrow-\infty$. Hence $u \leq u^{\ell} \leq u_{\mu^{+}}$for any $\ell \leq 0$. The sequence $\left\{g\left(u^{\ell}\right)\right\}_{\ell}$ is monotone nondecreasing with limit $g(u)$ when $\ell \rightarrow-\infty$, and there holds $g(u) \leq g\left(u^{\ell}\right) \leq g\left(u_{\mu^{+}}\right)$ for any $\ell \leq 0$. Since $g\left(u^{\ell}\right)$ is lower semicontinuous and $\sigma$-measurable, $g(u)$ shares the same properties.

Applying (3.37) to $\mu=\mu^{\ell}$ and $u=u^{\ell}$ gives

$$
\int_{\Omega}\left|u^{\ell}\right| d x+\int_{\Omega}\left|g\left(u^{\ell}\right)\right| \eta_{1} d \sigma \leq \int_{\Omega}\left|\mu^{\ell}\right| \eta_{1} d x
$$

Passing to the limit in the left-hand side using Fatou's lemma we obtain

$$
\int_{\Omega}|u| d x+\int_{\Omega}|g(u)| \eta_{1} d \sigma \leq \int_{\Omega}|\mu| \eta_{1} d x
$$

We deduce that $u \in L^{1}(\Omega)$ and $\rho g(u) \in L_{\sigma}^{1}(\Omega)$. We conclude as in Step 2 that $u$ is solution of (1.2).

Step 4: Proof of (3.17) and (3.18).

For $\ell<0<k$ we set $\mu_{k}^{\ell}=\sup \{\ell, \inf \{k, \mu\}\}$ and $\left(\mu^{\prime}\right)_{k}^{\ell}=\sup \left\{\ell, \inf \left\{k, \mu^{\prime}\right\}\right\}$, and denote by $u_{k}^{\ell}$ and $\left(u^{\prime}\right)_{k}^{\ell}$ the solution of $(1.2)$ with right-hand side $\mu_{k}^{\ell}$ and $\left(\mu^{\prime}\right)_{k}^{\ell}$. Then, by Corollary 3.8, for any $\zeta \in \mathbb{X}(\Omega)$ there holds

$$
-\int_{\Omega}\left|u_{k}^{\ell}-\left(u^{\prime}\right)_{k}^{\ell}\right| \Delta \zeta d x+\int_{\Omega}\left|g\left(u_{k}^{\ell}\right)-g\left(\left(u^{\prime}\right)_{k}^{\ell}\right)\right| \zeta d \sigma \leq \int_{\Omega} \operatorname{sign}_{0}\left(\mathrm{u}_{\mathrm{k}}^{\ell}-\left(\mathrm{u}^{\prime}\right)_{\mathrm{k}}^{\ell}\right)\left(\mu_{\mathrm{k}}^{\ell}-\left(\mu^{\prime}\right)_{\mathrm{k}}^{\ell}\right) \zeta \mathrm{dx} .
$$


Using the previous convergence theorem when $k \rightarrow \infty$ and then $\ell \rightarrow-\infty$, we derive (3.17). The proof of (3.18) is similar.

Remark. If it is not assumed that $g$ is nondecreasing, the above proof by monotonicity does not work. However the existence will follow from Theorem B if it is assumed that the extra assumptions in this theorem are satisfied: $\theta>N-q$ for some $q \in\left(1, \frac{N}{N-1}\right)$ and the growth assumptions of Theorem B.

\subsection{Diffuse case}

We recall that a measure $\mu$ is said to be diffuse with respect to the $c_{s, p}$-capacity

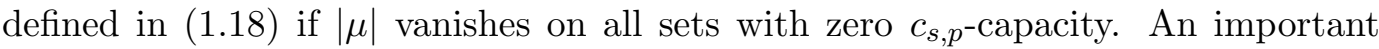
result due to Feyel and de la Pradelle [13] is the following:

Proposition 3.9 Let $\alpha>0$ and $1<p<\infty$. If $\lambda \in \mathfrak{M}_{b}^{+}(\Omega)$ does not charge sets with zero $c_{\alpha, p^{-}}$capacity, there exists an increasing sequence $\left\{\lambda_{n}\right\} \subset H^{-\alpha, p^{\prime}}(\Omega) \cap$ $\mathfrak{M}_{b}^{+}(\Omega), \lambda_{n}$ with compact support in $\Omega$, which converges to $\lambda$.

Proposition 3.10 Assume $\sigma \in \mathcal{M}_{\frac{N}{N-\theta}}^{+}$with $N \geq \theta>N-2$, and that $g: \mathbb{R} \mapsto \mathbb{R}$ is a continuous nondecreasing function vanishing at 0 . Then for any $\mu \in \mathfrak{M}_{b}^{+}(\Omega)$ diffuse with respect to the $c_{1,2}$-capacity there exists a unique very weak solution $u$ to (1.2).

Proof. According to Proposition 3.9, there exists an increasing sequence of nonnegative measures $\left\{\mu_{n}\right\}$ belonging to $W^{-1,2}(\Omega)$ and converging to $\mu$ and by Proposition 3.1, $\left\{u_{\mu_{n}}\right\}$ is a nondecreasing sequence of weak solutions of (1.2) with $\mu=\mu_{n}$. We claim that $u_{\mu_{n}} \uparrow u_{\mu}$ which is a very weak solution of (1.2). There holds,

$$
\int_{\Omega} u_{\mu_{n}} d x+\int_{\Omega} g\left(u_{\mu_{n}}\right) \eta_{1} d \sigma=\int_{\Omega} \eta_{1} d \mu_{n} \leq \int_{\Omega} \eta_{1} d \mu
$$

where $\eta_{1}$ is defined in (3.32). Since $u_{\mu_{n}} \geq 0, u_{\mu_{n}} \uparrow u$ and $g\left(u_{\mu_{n}}\right) \uparrow g(u)$. Since $u_{\mu_{n}}$ is $\sigma$-measurable by Proposition 3.1, $u$ is also $\sigma$-measurable. Hence $g(u)$ shares this measurability property since $g$ is continuous. Hence, by the monotone convergence theorem

$$
\int_{\Omega} u d x+\int_{\Omega} g(u) \eta_{1} d \sigma=\int_{\Omega} \eta_{1} d \mu .
$$

Furthermore $u_{\mu_{n}} \rightarrow u$ in $L^{1}(\Omega)$. Indeed it suffices to show that $\left\{u_{\mu_{n}}\right\}$ is uniformly equiintegrable which follows from $0 \leq \int_{\omega} u_{\mu_{n}} d x \leq \int_{\omega} u d x$ and the fact that $u \in$ $L^{1}(\Omega)$. We show in the same way that $\rho g\left(u_{\mu_{n}}\right) \rightarrow \rho g(u)$ in $L_{\sigma}^{1}(\Omega)$. This implies that $u=u_{\mu}$ is the very weak solution of (1.2). 


\subsection{Subcritical nonlinearities: proof of Theorem B.}

Lemma 3.11 Assume $N>2$ and $\sigma \in \mathcal{M}_{\frac{N}{N-\theta}}^{+}(\Omega)$ with $N \geq \theta>N-2$. If $\mu \in \mathfrak{M}_{b}(\Omega)$ and $\lambda \geq 0$, we set $E_{\lambda}[\mu]:=\{x \in \Omega: \mathbb{G}[|\mu|](x)>\lambda\}$. Then

$$
e_{\lambda}^{\sigma}(\mu):=\int_{E_{\lambda}[\mu]} d \sigma \leq c\|\mu\|_{\mathfrak{M}_{b}}^{\frac{\theta}{N-2}} \lambda^{-\frac{\theta}{N-2}} \quad \text { for all } \lambda>0 .
$$

Proof. It suffices to prove the result if $\mu \geq 0$. Indeed since $\mathbb{G}[|\mu|]=\mathbb{G}\left[\mu^{+}\right]+\mathbb{G}\left[\mu^{-}\right]$, we have $E_{\lambda}[\mu] \subset E_{\lambda / 2}\left[\mu^{+}\right] \cup E_{\lambda / 2}\left[\mu^{-}\right]$and thus $e_{\lambda}^{\sigma}(\mu) \leq e_{\lambda / 2}^{\sigma}\left(\mu^{+}\right)+e_{\lambda / 2}^{\sigma}\left(\mu^{+}\right)$. If the result holds for nonnegative measure, in particular for $\mu^{ \pm}$, then

$$
\begin{aligned}
\lambda^{\frac{\theta}{N-2}} e_{\lambda}^{\sigma}(\mu) & \leq c\left(\mu^{+}(\Omega)^{\frac{\theta}{N-2}}+\mu^{-}(\Omega)^{\frac{\theta}{N-2}}\right) \leq c\left(\mu^{+}(\Omega)+\mu^{-}(\Omega)\right)^{\frac{\theta}{N-2}} \\
& =c\|\mu\|_{\mathfrak{M}_{b}}^{\frac{\theta}{N-2}}
\end{aligned}
$$

Thus, we assume from now on that $\mu$ is nonnegative.

If $\mu=\delta_{a}$ for some $a \in \Omega$, then $\mathbb{G}\left[\delta_{a}\right](x) \leq c_{N}|x-a|^{2-N}$ so that $E_{\lambda}\left[\delta_{a}\right] \subset$ $B_{\left(\frac{c_{N}}{\lambda}\right)^{N-2}}(a)$. Since $\sigma \in \mathcal{M}_{\frac{N}{N-\theta}}^{+}(\Omega)$ it follows that

$$
e_{\lambda}^{\sigma}\left(\delta_{a}\right) \leq c \lambda^{-\frac{\theta}{N-2}}
$$

Let $E \subset \Omega$ be a Borel set. For any given $t>0$ there holds

$$
\int_{E} \mathbb{G}\left[\delta_{a}\right] d \sigma=\int_{E \cap E_{t}\left[\delta_{a}\right]} \mathbb{G}\left[\delta_{a}\right] d \sigma+\int_{E \cap E_{t}^{c}\left[\delta_{a}\right]} \mathbb{G}\left[\delta_{a}\right] d \sigma
$$

Clearly $\int_{E \cap E_{t}^{c}\left[\delta_{a}\right]} \mathbb{G}\left[\delta_{a}\right] d \sigma \leq t \sigma(E)$ and

$$
\int_{E \cap E_{t}\left[\delta_{a}\right]} \mathbb{G}\left[\delta_{a}\right] d \sigma \leq \int_{E_{t}\left[\delta_{a}\right]} \mathbb{G}\left[\delta_{a}\right] d \sigma \leq-\int_{t}^{\infty} s d e_{s}^{\sigma}\left(\delta_{a}\right) \leq c \frac{\theta t^{1-\frac{\theta}{N-2}}}{\theta+2-N},
$$

where the last inequality follows by integration by parts and the help of (3.40). Then

$$
\int_{E} \mathbb{G}\left[\delta_{a}\right] d \sigma \leq t \sigma(E)+c \frac{\theta t^{1-\frac{\theta}{N-2}}}{\theta+2-N} .
$$

Minimizing the right-hand side with respect to $t$, we infer

$$
\int_{E} \mathbb{G}\left[\delta_{a}\right] d \sigma \leq c \sigma(E)^{1-\frac{N-2}{\theta}} .
$$


We first suppose that $\mu=\sum_{j=1}^{\infty} \alpha_{j} \delta_{a_{j}}$ for some $\alpha_{j}>0$ and $a_{j} \in \Omega$. In particular $\sum_{j=1}^{\infty} \alpha_{j}=\|\mu\|_{\mathfrak{M}^{b}}$. Using Fubini's theorem and (3.41) we see that for any Borel set $E \subset \Omega$

$$
\int_{E} \mathbb{G}[\mu](x) d \sigma(x)=\sum_{j=1}^{\infty} \alpha_{j} \int_{E} \mathbb{G}\left[\delta_{a_{j}}(x)\right] d \sigma(x) \leq c \sigma(E)^{1-\frac{N-2}{\theta}}\|\mu\|_{\mathfrak{M}^{b}} .
$$

Taking in particular $E=E_{\lambda}[\mu]$ we obtain

$$
\lambda e_{\lambda}^{\sigma}(\mu) \leq \int_{E_{\lambda}[\mu]} \mathbb{G}[\mu](x) d \sigma(x) \leq c\left(e_{\lambda}^{\sigma}(\mu)\right)^{1-\frac{N-2}{\theta}}\|\mu\|_{\mathfrak{M}^{b}},
$$

which implies the claim. Notice that the constant $c$ in the right-hand side depends only on $N$ and $\|\sigma\|_{\mathcal{M}_{\frac{N}{N-\theta}}}$.

For a general nonnegative measure $\mu \in \mathfrak{M}_{b}(\Omega)$, we consider a sequence of nonnegative measures $\left\{\mu_{n}\right\} \subset \mathfrak{M}_{b}(\Omega)$ where each $\mu_{n}$ is a sum of Dirac masses as before and such that $\mu_{n} \rightarrow \mu$ weakly as $n \rightarrow \infty$. Then we have

$$
e_{\lambda}^{\sigma}\left(\mu_{n}\right):=\int_{E_{\lambda}\left[\mu_{n}\right]} d \sigma \leq c\left\|\mu_{n}\right\|_{\mathfrak{M}_{b}}^{\frac{\theta}{N-2}} \lambda^{-\frac{\theta}{N-2}}
$$

with $\|\mu\|_{\mathfrak{M}_{b}} \leq \liminf _{n \rightarrow \infty}\left\|\mu_{n}\right\|_{\mathfrak{M}_{b}}$. We thus need to prove that

$$
\liminf \int_{E_{\lambda}\left[\mu_{n}\right]} d \sigma \geq \int_{E_{\lambda}[\mu]} d \sigma
$$

We first observe that for any $t>0$ and $x \in \Omega$ the set $\{y \in \Omega: \mathbb{G}(x, y)>t\}$ is open (with $\mathbb{G}(x, x)=+\infty)$. It follows from [7][Thm 2.1] that $\liminf _{n \rightarrow \infty} \mu_{n}(\{\mathbb{G}(x, \cdot)>t\}) \geq$ $\mu(\{\mathbb{G}(x, \cdot)>t\})$. We can take the lim inf using Fatou's lemma in

$$
\int_{\Omega} \mathbb{G}(x, y) d \mu_{n}(y)=\int_{0}^{+\infty} \mu_{n}(\{\mathbb{G}(x, \cdot)>t\}) d t
$$

to derive

$$
\liminf _{n \rightarrow \infty} \mathbb{G}\left[\mu_{n}\right](x) \geq \int_{0}^{+\infty} \mu(\{G(x, \cdot)>t\}) d t=\int_{\Omega} G(x, y) d \mu(y)=\mathbb{G}[\mu](x) .
$$

We infer that for any $x \in \Omega$ such that $\chi_{E_{\lambda}(\mu)}(x)=1$ we have $\liminf _{n \rightarrow \infty} \mathbb{G}\left[\mu_{n}\right](x)>\lambda$, hence $\mathbb{G}\left[\mu_{n}\right](x)>\lambda$ for $n$ large enough. Thus $\chi_{E_{\lambda}\left(\mu_{n}\right)}(x)=1$ eventually, and then

$$
\liminf _{n \rightarrow \infty} \chi_{E_{\lambda}\left[\mu_{n}\right]}(x) \geq \chi_{E_{\lambda}[\mu]}(x) \quad \text { for all } x \in \Omega \text {. }
$$


The claim (3.43) follows by Fatou's lemma.

We are now in position to prove Theorem B.

Proof of Theorem B. We note that if $g$ is nondecreasing, uniqueness follows from estimate Lemma 3.5. Let $\left\{\eta_{n}\right\}$ be a sequence of mollifiers, $\mu_{n}=\mu * \eta_{n}$ and $u_{n} \in$ $W_{0}^{1,2}(\Omega)$ a minimizing weak solution of

$$
\begin{aligned}
-\Delta u_{n}+g\left(u_{n}\right) \sigma & =\mu_{n} & & \text { in } \Omega, \\
u_{n} & =0 & & \text { in } \partial \Omega,
\end{aligned}
$$

given by Proposition 3.1. We write $g(r)=g_{1}(r)+g_{2}(r)$ with $g_{1}=g \chi_{\left(-r_{0}, r_{0}\right)}$, $g_{2}=g \chi_{\left(-\infty-r_{0}\right] \cup\left[r_{0}, \infty\right)}$, and set $m=\sup \left\{g(r):-r_{0} \leq r \leq r_{0}\right\} \geq 0$ and $m^{\prime}=$ $\inf \left\{g(r):-r_{0} \leq r \leq r_{0}\right\} \leq 0$. Then

$$
-\mathbb{G}\left[\mu_{n}^{-}\right]-m \mathbb{G}[\sigma] \leq u_{n} \leq \mathbb{G}\left[\mu_{n}^{+}\right]-m^{\prime} \mathbb{G}[\sigma] .
$$

Since $\sigma \in \mathcal{M}_{p}^{+}(\Omega)$ for some $p>N / 2, \mathbb{G}[\sigma] \in C^{0, \alpha}(\bar{\Omega})$ by Lemma 2.2. Moreover $\mathbb{G}\left[\left|\mu_{n}\right|\right] \in C(\bar{\Omega})$ since $\left|\mu_{n}\right| \in C(\bar{\Omega})$. It follows that

$$
\left|u_{n}\right| \leq \mathbb{G}\left[\left|\mu_{n}\right|\right]+M \leq c_{n},
$$

where $M, c_{n} \geq 0$.

Since $u_{n} \in W_{0}^{1,2}(\Omega)$, its precise representative (that we identify with $u_{n}$ ) is defined $c_{1,2}$-quasi-everywhere, is $c_{1,2}$-continuous and

$$
u_{n}(x)=\lim _{r \rightarrow 0} \frac{1}{\left|B_{r}(x)\right|} \int_{B_{r}(x)} u_{n}(y) d y
$$

for any $y \in \Omega \backslash E_{n}$ with $c_{1,2}\left(E_{n}\right)=0$ (see [2]). It follows that $\left|u_{n}\right| \leq c_{n}$ in $E:=\cup E_{n}$. Note that $c_{1,2}(E)=0$ so that $\sigma(E)=0$ by Lemma 3.3. Hence $\left|u_{n}\right| \leq c_{n} \sigma$-almost everywhere, $g\left(u_{n}\right) \in L_{\sigma}^{\infty}(\Omega)$, and therefore $g\left(u_{n}\right) \sigma \in \mathcal{M}_{\frac{N}{N-\theta}}^{+}(\Omega)$. We can then apply Corollary 3.8 to obtain, for any $\zeta \in \mathbb{X}_{+}(\Omega)$, that

$$
-\int_{\Omega}|u|_{n} \Delta \zeta d x+\int_{\Omega} \operatorname{sign}_{0}\left(u_{n}\right) g\left(u_{n}\right) \zeta d \sigma \leq \int_{\Omega} \operatorname{sign}_{0}\left(u_{n}\right) \zeta \mu_{n} d x,
$$

which implies

$$
-\int_{\Omega}|u|_{n} \Delta \zeta d x+\int_{\Omega}\left|g_{2}\left(u_{n}\right)\right| \zeta d \sigma \leq \int_{\Omega} \operatorname{sign}_{0}\left(u_{n}\right) \zeta \mu_{n} d x+c \int_{\Omega} \zeta d \sigma .
$$

We take $\zeta=\eta_{1}$ and obtain

$$
\begin{aligned}
\int_{\Omega}\left|u_{n}\right| d x+\int_{\Omega}\left|g_{2}\left(u_{n}\right)\right| \eta_{1} d \sigma & \leq \int_{\Omega}\left|\mu_{n}\right| \eta_{1} d x+c \\
& \leq \int_{\Omega} \eta_{1} d|\mu|+c=c^{\prime}
\end{aligned}
$$


so that $\left\{u_{n}\right\}$ is bounded in $L^{1}(\Omega)$. We also have from Corollary 3.8 that

$$
\int_{\Omega} \operatorname{sign}_{0}\left(u_{n}\right) g\left(u_{n}\right) d \sigma \leq C \int_{\Omega}\left|\mu_{n}\right| \rho d x
$$

and so

$$
\int_{\Omega}\left|g_{2}\left(u_{n}\right)\right| d \sigma \leq C \int_{\Omega}\left|\mu_{n}\right| d x+\int_{\Omega}\left|g_{1}\left(u_{n}\right)\right| d \sigma \leq C
$$

with $C$ independent of $n$. We deduce that the sequence of measures $\left\{g\left(u_{n}\right)\right\}$ is bounded.

By the standard regularity estimates, the sequence $\left\{u_{n}\right\}$ is bounded in $W^{1, q}(\Omega)$, $q<\frac{N}{N-1}$. Then there exists $u \in W^{1, q}(\Omega), q<\frac{N}{N-1}$, such that, up to a subsequence, $u_{n} \rightarrow u$ in $L^{1}(\Omega)$ and also pointwise in $\Omega \backslash E$ where $c_{1, q}(E)=0$. We fix $q \in\left(1, \frac{N}{N-1}\right)$ such that $\theta>N-q$. In view of Lemma 3.3, $\sigma(E)=0$ so that $g\left(u_{n}\right) \rightarrow g(u) \sigma$-almost everywhere. Applying Fatou's lemma in (3.48) gives that $g(u) \in L_{\sigma}^{1}(\Omega)$.

In order to prove the uniform integrability of $\left\{g\left(u_{n}\right)\right\}$ for the measure $\sigma$ we can assume that $\left|g_{2}\right| \leq \tilde{g}$ with a function satisfying (1.8) still denoted by $\tilde{g}$ and let $E \subset \Omega$ be a Borel set. Then

$$
\begin{aligned}
\int_{E}\left|g_{2}\left(u_{n}\right)\right| d \sigma & \leq \int_{E \cap\left\{\left|u_{n}\right| \leq t\right\}}\left|g_{2}\left(u_{n}\right)\right| d \sigma+\int_{E \cap\left\{\left|u_{n}\right|>t\right\}}\left|g_{2}\left(u_{n}\right)\right| d \sigma \\
& \leq \tilde{g}(t) \int_{E} d \sigma+\int_{\left\{\left|u_{n}\right|>t\right\}} \tilde{g}\left(\left|u_{n}\right|\right) d \sigma .
\end{aligned}
$$

Then we estimate the second integral in the right-hand side: for $\lambda>M$ we set

$$
S_{n}(\lambda)=\left\{x \in \Omega:\left|u_{n}(x)\right|>\lambda\right\} \quad \text { and } b_{n}^{\sigma}(\lambda)=\int_{S_{n}(\lambda)} d \sigma
$$

In view of (3.45) we have $\left|u_{n}\right| \leq \mathbb{G}\left(\left|\mu_{n}\right|\right)+M$ so that $S_{n}(\lambda) \subset E_{\lambda-M}\left[\mu_{n}\right]$. Hence $b_{n}^{\sigma}(\lambda) \leq e_{\lambda-M}^{\sigma}\left(\left|\mu_{n}\right|\right)$. This implies

$$
\begin{aligned}
\int_{\left\{\left|u_{n}\right|>t\right\}} \tilde{g}\left(\left|u_{n}\right|\right) d \sigma & =-\int_{t}^{\infty} \tilde{g}(\lambda) d b_{n}^{\sigma}(\lambda) \\
& \leq \int_{t}^{\infty} b_{n}^{\sigma}(\lambda) d \tilde{g}(\lambda) \\
& \leq \int_{t}^{\infty} e_{\lambda-M}^{\sigma}\left(\left|\mu_{n}\right|\right) d \tilde{g}(\lambda) .
\end{aligned}
$$

Using (3.39) we obtain

$$
\begin{aligned}
\int_{\left\{\left|u_{n}\right|>t\right\}} \tilde{g}\left(\left|u_{n}\right|\right) d \sigma & \leq c\|\mu\|_{\mathfrak{M}^{b}}^{\frac{\theta}{N-2}} \int_{t}^{\infty}(\lambda-M)^{-\frac{\theta}{N-2}} d \tilde{g}(\lambda) \\
& \leq \frac{c \theta}{N-2} \int_{t}^{\infty}(\lambda-M)^{-\frac{\theta}{N-2}-1} \tilde{g}(\lambda) d \lambda
\end{aligned}
$$


In view of assumption (1.8), given $\epsilon>0$ we fix $t>M$ such that

$$
\frac{c \theta}{N-2} \int_{t}^{\infty}(\lambda-M)^{-\frac{\theta}{N-2}-1} \tilde{g}(\lambda) d \lambda \leq \frac{\varepsilon}{2}
$$

Then, setting $\delta=\frac{\epsilon}{2 \tilde{g}(t)}$, we deduce

$$
\int_{E} d \sigma \leq \delta \Longrightarrow \int_{E}\left|g_{2}\left(u_{n}\right)\right| d \sigma \leq \varepsilon
$$

Since $g_{1}$ is bounded, this implies that $\left\{g\left(u_{n}\right)\right\}$ is uniformly integrable in $L_{\sigma}^{1}(\Omega)$. Since we already know that $g\left(u_{n}\right) \rightarrow g(u) \sigma$-almost everywhere, it follows by Vitali's convergence theorem that $g\left(u_{n}\right) \rightarrow g(u)$ in $L_{\sigma}^{1}(\Omega)$. Taking $\zeta \in \mathbb{X}(\Omega)$ and letting $n \rightarrow \infty$ in the equality

$$
-\int_{\Omega} u_{n} \Delta \zeta d x+\int_{\Omega} g\left(u_{n}\right) \zeta d \sigma=\int_{\Omega} \zeta d \mu_{n}
$$

yields the result.

\section{The 2-D case}

In this section $\Omega$ is a bounded $C^{2}$ planar domain. The next result is the $2-\mathrm{D}$ version of Lemma 3.11 .

Lemma 4.1 Assume $N=2$ and $\sigma \in \mathcal{M}_{\frac{2}{2-\theta}}^{+}(\Omega)$ with $2 \geq \theta>0$. If $\mu \in \mathfrak{M}^{b}(\Omega)$ and $\lambda \geq 0$, we set $E_{\lambda}[\mu]:=\{x \in \Omega: \mathbb{G}[|\mu|](x)>\lambda\}$. Then

$$
e_{\lambda}^{\sigma}(\mu):=\int_{E_{\lambda}[\mu]} d \sigma \leq|\Omega|_{\sigma} e^{1-\frac{\lambda}{\gamma\|\mu\|_{\mathfrak{M}^{b}}}} \quad \text { for all } \lambda>0,
$$

for some $\gamma=\gamma(\theta$, $\operatorname{diam}(\Omega))>0$

Proof. If $\mu=\delta_{a}$ for some $a \in \Omega$, one has $0 \leq \mathbb{G}\left[\delta_{a}\right](x) \leq \frac{1}{2 \pi} \ln \left(\frac{d_{\Omega}}{|x-a|}\right)$ where $d_{\Omega}=\operatorname{diam}(\Omega)$. Hence

$$
E_{\lambda}\left[\delta_{a}\right] \subset B_{d_{\Omega} e^{-2 \pi \lambda}} \Longrightarrow e_{\lambda}^{\sigma}\left(\delta_{a}\right)=\int_{E_{\lambda}\left[\delta_{a}\right]} d \sigma \leq c d_{\Omega}^{\theta} e^{-2 \theta \pi \lambda}
$$

Let $E \subset \Omega$ be a Borel set, $\int_{E} d \sigma=|E|_{\sigma}$ and $t>0$, then, as in Lemma 3.11,

$$
\begin{aligned}
\int_{E} \mathbb{G}\left[\delta_{a}\right] d \sigma & \leq t \int_{E} d \sigma-\int_{t}^{\infty} s d e_{s}^{\sigma}\left(\delta_{a}\right) \\
& \leq t|E|_{\sigma}+c d_{\Omega}^{\theta}\left(t+\frac{1}{2 \pi \theta}\right) e^{-2 \theta \pi t}
\end{aligned}
$$


If we choose $e^{-2 \theta \pi t}=\frac{|E|_{\sigma}}{|\Omega|_{\sigma}}$ we infer

$$
\int_{E} \mathbb{G}\left[\delta_{a}\right] d \sigma \leq \gamma|E|_{\sigma}\left(\ln \left(\frac{|\Omega|_{\sigma}}{|E|_{\sigma}}\right)+1\right) .
$$

For proving (3.39) we can assume that $\mu \geq 0$. Then there exists $\alpha_{j}>0$ and $a_{j} \in \Omega$ such that

$$
\mu=\sum_{j=1}^{\infty} \alpha_{j} \delta_{a_{j}} \Longrightarrow \sum_{j=1}^{\infty} \alpha_{j}=\|\mu\|_{\mathfrak{M}^{b}}
$$

Hence, for any Borel set $E \subset \Omega$,

$$
\int_{E} \mathbb{G}[\mu](x) d \sigma(x)=\sum_{j=1}^{\infty} \alpha_{j} \int_{E} \mathbb{G}\left[\delta_{a_{j}}(x)\right] d \sigma(x) \leq \gamma|E|_{\sigma}\left(\ln \left(\frac{|\Omega|_{\sigma}}{|E|_{\sigma}}\right)+1\right)\|\mu\|_{\mathfrak{M}^{b}} .
$$

If $E=E_{\lambda}[\mu]$ we infer

$$
\lambda e_{\lambda}^{\sigma}(\mu) \leq \gamma e_{\lambda}^{\sigma}(\mu)\left(\ln \left(\frac{|\Omega|_{\sigma}}{e_{\lambda}^{\sigma}(\mu)}\right)+1\right)\|\mu\|_{\mathfrak{M}^{b}},
$$

which implies the claim.

Theorem 4.2 Assume $N=2, \sigma \in \mathcal{M}_{\frac{2}{2-\theta}}^{+}(\Omega)$ with $2 \geq \theta>0$ and $g: \mathbb{R} \mapsto \mathbb{R} a$ continuous function satisfying (1.1). If $a_{\infty}(g)=a_{-\infty}(g)=0$, for any $\mu \in \mathfrak{M}_{b}(\Omega)$ problem (1.2) admits a very weak solution.

Proof. Let $g^{*}$ be the monotone nondecreasing hull of $g$ defined by (1.11). If $m=$ $\sup \left\{g(r):-r_{0} \leq r \leq r_{0}\right\}$ and $m^{\prime}=\inf \left\{g(r):-r_{0} \leq r \leq r_{0}\right\}$ then $g \leq g^{*}+m$ on $\mathbb{R}_{+}$and $g^{*}+m^{\prime} \leq g$ on $\mathbb{R}_{-}$. If $\left\{\eta_{n}\right\}$ is a sequence of mollifiers and $\mu=\mu^{+}-\mu^{-}$, we set $\mu_{n}^{+}=\mu^{+} * \eta_{n}, \mu_{n}^{-}=\mu_{-} * \eta_{n}, \mu_{n}=\mu_{n}^{+}=-\mu_{n}^{-}$and denote by $u_{n}$ the very weak solution of

$$
\begin{aligned}
-\Delta u_{n}+g\left(u_{n}\right) \sigma & =\mu_{n} & & \text { in } \Omega \\
u_{n} & =0 & & \text { on } \partial \Omega .
\end{aligned}
$$

Since $\left\|\mu_{n}\right\|_{L^{1}} \leq\|\mu\|_{\mathfrak{M}_{b}}$, there holds by Proposition 3.2 ,

$$
\left\|u_{n}\right\|_{L^{1}}+\left\|\rho g\left(u_{n}\right)\right\|_{L_{\sigma}^{1}} \leq c\|\mu\|_{\mathfrak{M}_{b}}+M
$$

and by Lemma 2.1,

$$
\left\|u_{n}\right\|_{B M O}+\left\|\nabla u_{n}\right\|_{L^{2, \infty}} \leq c\left(\|\mu\|_{\mathfrak{M}_{b}}+\left\|\rho g\left(u_{n}\right)\right\|_{L_{\sigma}^{1}}\right) \leq c^{\prime}\|\mu\|_{\mathfrak{M}_{b}} .
$$

Again, there exists a set $E$ with $c_{1, q}(E)=0$ for any $q \leq 2-\theta$ such that $u_{n}(x) \rightarrow u(x)$ for all $x \in \Omega \backslash E$, hence $u_{n}(x) \rightarrow u(x)$ and $g\left(u_{n}(x)\right) \rightarrow g(u(x)) d \sigma$-almost everywhere 
in $\Omega$. This implies that $g(u)$ is $\sigma$-measurable. In order to conclude we have to prove that $g\left(u_{n}\right) \rightarrow g(u)$ in $L_{\sigma}^{1}(\Omega)$. Estimate (4.1) is valid, hence, for any $t>0$,

$$
\tau_{n}(t)=\int_{\left\{\left|u_{n}(x)\right|>t\right\}} d \sigma \leq e_{t-M}^{\sigma}\left[\mu_{n}^{+}\right]+e_{t-M^{\prime}}^{\sigma}\left[\mu_{n}^{-}\right] \leq c e^{-\frac{t}{\gamma\|\mu\|_{\mathfrak{M}}}}
$$

by Lemma 4.1. Since

$$
\left|g\left(u_{n}\right)\right| \leq\left(g_{+}^{*}\left(u_{n}\right)-g_{-}^{*}\left(u_{n}\right)\right)+m-m^{\prime},
$$

we have that

$$
\begin{aligned}
\int_{E}\left|g\left(u_{n}\right)\right| d \sigma & \leq \int_{E} g_{+}^{*}\left(u_{n}\right) d \sigma-\int_{E} g_{-}^{*}\left(u_{n}\right) d \sigma+\left(m-m^{\prime}\right)|E|_{\sigma} \\
& \leq-\int_{t}^{\infty} g_{+}^{*}(s) d\left|\left\{u_{n}>s\right\}\right|_{\sigma}+\int_{-\infty}^{-t} g_{-}^{*}(s) d\left|\left\{u_{n}<s\right\}\right|_{\sigma}+\left(m-m^{\prime}\right)|E|_{\sigma} \\
& \leq-\int_{t}^{\infty}\left(g_{+}^{*}(s)-g_{-}^{*}(-s)\right) d \tau_{n}(s)+\left(g_{+}^{*}(t)-g_{-}^{*}(-t)+m-m^{\prime}\right)|E|_{\sigma} .
\end{aligned}
$$

By integration by parts,

$$
\begin{aligned}
& -\int_{t}^{\infty}\left(g_{+}^{*}(s)-g_{-}^{*}(-s)\right) d \tau_{n}(s)=\left(g_{+}^{*}(t)-g_{-}^{*}(-t)\right) \tau_{n}(t)+\int_{t}^{\infty} \tau_{n}(s) d\left(g_{+}^{*}(s)-g_{-}^{*}(-s)\right) \\
& \leq\left(g_{+}^{*}(t)-g_{-}^{*}(-t)\right)\left(\tau_{n}(t)-c e^{-\frac{t}{\gamma\|\mu\|_{\mathfrak{M}^{b}}}}\right) \\
& +\frac{c}{\gamma\|\mu\|_{\mathfrak{M}^{b}}} \int_{t}^{\infty} e^{-\frac{s}{\gamma\|\mu\|_{\mathfrak{M}^{b}}}}\left(g_{+}^{*}(s)-g_{-}^{*}(-s)\right) d s \\
& \leq \frac{c}{\gamma\|\mu\|_{\mathfrak{M}^{b}}} \int_{t}^{\infty} e^{-\frac{s}{\gamma\|\mu\|_{\mathfrak{M}^{b}}}}\left(g_{+}^{*}(s)-g_{-}^{*}(-s)\right) d s .
\end{aligned}
$$

By assumption the integral on the right-hand side is convergent. We end the proof as in Theorem B, first by fixing $t$ large enough and then $|E|_{\sigma}$ small enough, and we derive the uniform integrability of $\left\{g\left(u_{n}\right)\right\}$.

A similar result holds when $g$ has nonzero order of growth at infinty.

Theorem 4.3 Assume $N=2, \sigma \in \mathcal{M}_{\frac{2}{2-\theta}}^{+}(\Omega)$ with $2 \geq \theta>0$ and $g: \mathbb{R} \mapsto \mathbb{R} a$ continuous function satisfying (1.1). If $0<a_{\infty}(g)<\infty$ and $-\infty<a_{-\infty}(g)<0$, there exists $\delta>0$ such that for any $\mu \in \mathfrak{M}_{b}(\Omega)$ satisfying $\|\mu\|_{\mathfrak{M}_{b}} \leq \delta$ problem (1.2) admits a very weak solution.

Proof. The proof is a straightforward adaptation of the previous one. The choice of $\delta$ is such that

$$
\|\mu\|_{\mathfrak{M}_{b}} \leq \delta<\frac{1}{\gamma} \sup \left\{\frac{1}{a_{\infty}(g)},-\frac{1}{a_{-\infty}(g)}\right\}
$$

and the conclusion follows from (4.7). 


\section{The supercritical case}

\subsection{Proof of Theorem D}

Proof of assertion $I$. For $k>0$ set $g_{k}(r)=\max \{g(-k), \min \{g(k), g(r)\}\}$ and denote by $u_{k}$ the very weak solution of

$$
\begin{aligned}
-\Delta u+g_{k}(u) \sigma & =\mu & & \text { in } \Omega \\
u & =0 & & \text { on } \partial \Omega,
\end{aligned}
$$

which exists by Theorem B. It follows from the proof of Theorem B (see (3.48) with $g=g_{2}$ and $\left.g_{1}=0\right)$ that

$$
\int_{\Omega}\left|g_{k}\left(u_{k}\right)\right| d \sigma \leq C
$$

where the constant $C$ depends only on $\Omega$ and $|\mu|(\Omega)$. Thus the sequence of measures $\left\{g_{k}\left(u_{k}\right) \sigma\right\}$ is bounded. This implies that $\left\{u_{k}\right\}$ is bounded in $W^{1, q}(\Omega), q<\frac{N}{N-1}$, and thus that, up to a subsequence, it converges in $L^{1}(\Omega)$ to some $u \in W^{1, q}(\Omega)$, $q<\frac{N}{N-1}$. We can also assume that the convergence holds pointwise except on a set $E$ with zero $c_{1, q}$-capacity, which in turn is $\sigma$-negligible by Lemma 3.3 if we fix

$q \in\left(1, \frac{N}{N-1}\right)$ such that $\theta>N-q$. We also have that $u$ is finite but on a set with zero $c_{1, q}$-capacity hence $\sigma$-negligible, therefore

$$
g_{k}\left(u_{k}\right) \rightarrow g(u) \quad \sigma \text {-almost everywhere. }
$$

Applying Fatou's lemma in (5.2) yields $g(u) \in L_{\sigma}^{1}(\Omega)$.

By the maximum principle

$$
-\mathbb{G}[|\mu|] \leq u_{k} \leq \mathbb{G}[|\mu|]
$$

hence

$$
g(-\mathbb{G}[|\mu|]) \leq g_{k}\left(u_{k}\right) \leq g(\mathbb{G}[|\mu|]),
$$

since $g$ is nondecreasing.

Because of assumption (1.13) and in view of (5.4), we infer from Lebesgue dominated convergence that $\rho g_{k}\left(u_{k}\right) \rightarrow \rho g(u)$ in $L_{\sigma}^{1}(\Omega)$. Thus we can pass to the limit in weak formulation of (5.1) with any $\zeta \in \mathbb{X}(\Omega)$.

Proof of assertion II. We first notice that if $g$ is nondecreasing, vanishes at 0 and satisfies (1.14), then the function $g_{k}$ defined above also satisfies (1.14) with the same constants $a$ and $b$. We assume first that $\mu=\mu_{r}+\mu_{s}$ is nonnegative and we set $\mu_{r}^{n}=\mu_{r} * \eta_{n}$ where $\left\{\eta_{n}\right\}$ is a sequence of mollifiers. Let $u_{k}^{n}$ be the solution of (5.1) with right-hand side $\mu_{r}^{n}+\mu_{s}$ and $v_{k}^{n}$ the one of (5.1) with right-hand side $\mu_{r}^{n}$ (in both 
cases existence and uniqueness follows from Theorem B). Then $0 \leq u_{k}^{n} \leq v_{k}^{n}+\mathbb{G}\left[\mu_{s}\right]$, $v_{k}^{n} \geq 0$ and $\mathbb{G}\left[\mu_{s}\right] \geq 0$. Since $g$ is non-decreasing, we deduce with (1.14) that

$$
0 \leq g_{k}\left(u_{k}^{n}\right) \leq g_{k}\left(v_{k}^{n}+\mathbb{G}\left[\mu_{s}\right]\right) \leq a\left(g_{k}\left(v_{k}^{n}\right)+g_{k}\left(\mathbb{G}\left[\mu_{s}\right]\right)\right)+b
$$

Since

$$
\left\|v_{k}^{n}\right\|_{L^{1}}+\left\|\rho g_{k}\left(v_{k}^{n}\right)\right\|_{L_{\sigma}^{1}} \leq c\left\|\mu_{r}^{n}\right\|_{\mathfrak{M}_{b}} \leq c\|\mu\|_{\mathfrak{M}_{b}},
$$

up to subsequences, the sequences $\left\{v_{k}^{n}\right\}$ and $\left\{u_{k}^{n}\right\}$ converge in $L^{1}(\Omega)$ to some $v^{n} \in$ $L^{1}(\Omega)$ and $u^{n}$ such that $\nabla v^{n}, \nabla u^{n} \in L^{q}(\Omega)$ for any $q<\frac{N}{N-1}$ when $k \rightarrow \infty$. As in I, $\left\{g_{k}\left(v_{k}^{n}\right)\right\}$ and $\left\{g_{k}\left(u_{k}^{n}\right)\right\}$ converge in $L_{\sigma}^{1}(\Omega)$ to $\left\{g\left(v^{n}\right)\right\}$ and $\left\{g\left(u^{n}\right)\right\}$ respectively. Furthermore $v^{n}$ and $u^{n}$ satisfy

$$
\begin{aligned}
-\Delta v^{n}+g\left(v^{n}\right) \sigma & =\mu_{r}^{n} & & \text { in } \Omega \\
v^{n} & =0 & & \text { on } \partial \Omega,
\end{aligned}
$$

and

$$
\begin{aligned}
-\Delta u^{n}+g\left(u^{n}\right) \sigma & =\mu_{s}+\mu_{r}^{n} & & \text { in } \Omega \\
u^{n} & =0 & & \text { on } \partial \Omega,
\end{aligned}
$$

respectively and $0 \leq u^{n} \leq v^{n}+\mathbb{G}\left[\mu_{s}\right]$. As in the proof of Proposition 3.2, $v^{n} \rightarrow v$ in $L^{1}(\Omega)$ and $\rho g\left(v^{n}\right) \rightarrow \rho g(v)$ in $L_{\sigma}^{1}(\Omega)$ as $n \rightarrow \infty$, and $v$ is a very weak solution of

$$
\begin{aligned}
-\Delta v+g(v) \sigma & =\mu_{r} & & \text { in } \Omega \\
v & =0 & & \text { on } \partial \Omega .
\end{aligned}
$$

As above $\left\{u^{n}\right\}$ converge in $L^{1}(\Omega)$ to some $u \in L^{1}(\Omega)$ (always up to some subsequence), there holds $u \leq v+\mathbb{G}\left[\mu_{s}\right]$ and $g\left(u^{n}\right) \rightarrow g(u) \sigma$-almost everywhere in $\Omega$ since the uniform bound on $\left\|\nabla u_{n}\right\|_{L^{\frac{N}{N-1}}, \infty}$ holds. Furthermore

$$
0 \leq g\left(u^{n}\right) \leq a\left(g\left(v^{n}\right)+g\left(\mathbb{G}\left[\mu_{s}\right]\right)\right)+b \Longrightarrow 0 \leq g(u) \leq a\left(g(v)+g\left(\mathbb{G}\left[\mu_{s}\right]\right)\right)+b,
$$

and since $g\left(v^{n}\right) \rightarrow g(v)$ in $L_{\sigma}^{1}(\Omega)$, the sequence $\left\{g\left(u^{n}\right)\right\}$ is uniformly integrable in $L_{\sigma}^{1}(\Omega)$. Again this implies that $g\left(u^{n}\right) \rightarrow g(u)$ in $L_{\sigma}^{1}(\Omega)$ and $u$ is a very weak solution of (1.2). If $\mu$ is signed measure, we construct successively the solutions $u_{k}^{n}, \bar{u}_{k}^{n}$ and $\underline{u}_{k}^{n}$ of (5.1) with right-hand side $\mu_{r}^{n}+\mu_{s},\left|\mu_{r}^{n}\right|+\left|\mu_{s}\right|$ and $-\left|\mu_{r}^{n}\right|-\left|\mu_{s}\right|$ respectively, and the solutions $\bar{v}_{k}^{n}$ and $\underline{v}_{k}^{n}$ of (5.1) with right-hand side $\left|\mu_{r}^{n}\right|$ and $-\left|\mu_{r}^{n}\right|$ respectively. Then $\underline{v}_{k}^{n}-\mathbb{G}\left[\mu_{s}\right] \leq u_{k}^{n} \leq \bar{v}_{k}^{n}+\mathbb{G}\left[\mu_{s}\right]$ which implies by (1.15)

$$
a\left(g_{k}\left(\underline{v}_{k}^{n}\right)+g_{k}\left(-\mathbb{G}\left[\mu_{s}\right]\right)\right)+b \leq g_{k}\left(u_{k}^{n}\right) \leq a\left(g_{k}\left(\bar{v}_{k}^{n}\right)+g_{k}\left(\mathbb{G}\left[\mu_{s}\right]\right)\right)+b .
$$

Using the same estimates as above we conclude that $\lim _{n \rightarrow \infty} \lim _{k \rightarrow \infty} u_{k}^{n}=u$ exists in $L^{1}(\Omega)$, that $\lim _{n \rightarrow \infty} \lim _{k \rightarrow \infty} g_{k}\left(u_{k}^{n}\right)=g(u)$ holds $\sigma$ almost everywhere in $\Omega$ and in $L_{\sigma}^{1}(\Omega)$, which ends the proof. 


\subsection{Reduced measures}

We adapt here some of the results in [9] which turn out to be useful tools in our framework.

Lemma 5.1 Let $\sigma \in \mathcal{M}_{\frac{N}{N-\theta}}^{+}(\Omega)$ with $N \geq \theta>N-\frac{N}{N-1}$ and $g$ be nondecreasing satisfying (1.1). Assume $\left\{\mu_{n}\right\} \subset \mathfrak{M}_{b}^{+}(\Omega)$ is an increasing sequence of good measures for problem (1.2) converging to $\mu \in \mathfrak{M}_{b}^{+}(\Omega)$. Then $\mu$ is a good measure.

Proof. Let $u_{\mu_{n}}$ be the solutions of (1.2) with right-hand side $\mu_{n}$ then for any $n, k \in \mathbb{N}$, $k \geq n$, we have since $u_{0} \in C^{\alpha}(\bar{\Omega})$,

$$
-m \leq u_{0} \leq u_{\mu_{n}} \leq u_{\mu_{k}}
$$

for some $m \geq 0$ and then

$$
g(-m) \leq g\left(u_{0}\right) \leq g\left(u_{\mu_{n}}\right) \leq g\left(u_{\mu_{k}}\right) .
$$

We use $\zeta:=\left(\eta_{1}+\epsilon\right)^{\alpha}-\epsilon^{\alpha}$ as a test-function in the very weak formulation of the equation satisfied by $u_{\mu_{n}}-u_{0}$ as in the proof of (3.30); then, recalling that $-\Delta \zeta \geq 0$, we obtain that

$$
\int_{\Omega}\left(g\left(u_{\mu_{n}}\right)-g\left(u_{0}\right)\right)\left(\left(\eta_{1}+\epsilon\right)^{\alpha}-\epsilon^{\alpha}\right) d \sigma \leq \int_{\Omega}\left(\eta_{1}+\epsilon\right)^{\alpha} d \mu_{n} \leq C \mu_{n}(\Omega) \leq C \mu(\Omega),
$$

where $C$ is independent of $n$. Letting successively $\epsilon \rightarrow 0$ and $\alpha \rightarrow 0$ we obtain

$$
0 \leq \int_{\Omega}\left(g\left(u_{\mu_{n}}\right)-g\left(u_{0}\right)\right) d \sigma \leq C
$$

Hence $\left\{u_{\mu_{n}}\right\}$ is bounded in $W_{0}^{1, q}(\Omega)$ for any $q<\frac{N}{N-1}$. Thus there exists $u \in W_{0}^{1, q}(\Omega)$, $q<\frac{N}{N-1}$, such that $u_{\mu_{n}} \uparrow u$ in $L^{1}(\Omega)$ and pointwise but for a set $E$ with zero $c_{1, q^{-}}$ capacity. Since $\theta>N-\frac{N}{N-1}$ we can find some $q<\frac{N}{N-1}$ such that $\theta>N-q$. It then follows from Lemma 3.3 that $\sigma(E)=0$. Thus $g\left(u_{\mu_{n}}\right) \uparrow g(u) \sigma$-almost everywhere. Fatou's lemma yields $\int_{\Omega}\left(g(u)-g\left(u_{0}\right)\right) d \sigma \leq C$, thus $g(u) \in L_{\sigma}^{1}(\Omega)$. By the dominated convergence theorem, $g\left(u_{\mu_{n}}\right) \rightarrow g(u)$ in $L_{\sigma}^{1}$. We can then pass to the limit in the equation satisfied by $u_{\mu_{n}}$ to obtain that $u=u_{\mu}$.

Proposition 5.2 Assume $\sigma$ and $g$ satisfy the assumptions of Lemma 5.1. Consider the set

$$
Z=\left\{x \in \Omega: \int_{\Omega} \mathbb{G}(x, y)^{q} \rho(y) d \sigma(y)=\infty\right\}
$$

If $\mu \in \mathfrak{M}_{b}^{+}(\Omega)$ is such that $\mu(Z)=0$ then $\mu$ is good. 
Proof. We adapt to our case the proof of [30][Thm 3.10]. Consider the sets

$$
C_{n}=\left\{x \in \Omega: \int_{\Omega} \mathbb{G}(x, y)^{q} \rho(y) d \sigma(y) \leq n\right\}, \quad n=1,2, \ldots
$$

Since the function $x \rightarrow \int_{\Omega} \mathbb{G}(x, y)^{q} \rho(y) d \sigma(y)$ is lsc (by Fatou's lemma) the sets $C_{n}$ are closed. Moreover $C_{n} \subset C_{n+1}$ and $\bigcup_{n} C_{n}=\Omega \backslash Z$. Define $\mu_{n}:=1_{C_{n}} \mu$ i.e. $\mu_{n}$ is the measure $\mu$ restricted to $C_{n}$. Then each $\mu_{n}$ satisfies (1.13). Indeed

$$
\begin{aligned}
\int_{\Omega} \mathbb{G}\left[\left|\mu_{n}\right|\right]^{q} \rho d \sigma & \leq \mu_{n}(\Omega)^{q-1} \int_{\Omega} \int_{\Omega} \mathbb{G}(x, y)^{q-1} d \mu_{n}(x) d \sigma(y) \\
& \leq \mu(\Omega)^{q-1} \int_{C_{n}}\left(\int_{\Omega} \mathbb{G}(x, y)^{q-1} d \sigma(y)\right) d \mu(x) \\
& \leq n \mu(\Omega)^{q} .
\end{aligned}
$$

It follows from Theorem $\mathrm{D}$ that $\mu_{n}$ is good. Since $0 \leq \mu_{n} \uparrow \mu$ we deduce from Lemma 5.1 that $\mu$ is good.

Lemma 5.3 Assume $\sigma$ and $g$ satisfy the assumptions of Lemma 5.1.

I- If $\mu \in \mathfrak{M}_{b}^{+}(\Omega)$ is a good measure, any $\nu \in \mathfrak{M}_{b}^{+}(\Omega)$ such that $\nu \leq \mu$ is a good measure.

II- Let $\mu, \mu^{\prime} \in \mathfrak{M}_{b}^{+}(\Omega)$. If $\mu$ and $-\mu^{\prime}$ are good measures, any $\nu \in \mathfrak{M}_{b}(\Omega)$ such that $-\mu^{\prime} \leq \nu \leq \mu$ is a good measure.

Proof. Step 1. Assume $\mu \in \mathfrak{M}_{b}^{+}(\Omega)$ is a good measure. For $k>0$ define $g_{k}$ by $g_{k}(r)=$ $\max \{g(-k), \min \{g(k), g(r)\}\}$, and denote by $u_{k, \mu}$ the solution of (5.1), which exists by Theorem B, and by $u_{\mu}$ the solutions of (1.2). Then $-m \leq u_{0} \leq \min \left\{u_{\mu}, u_{k, \mu}\right\}$. If $k>m$, then $g_{k}\left(u_{k, \mu}\right)=\min \left\{g(k), g\left(u_{k, \mu}\right)\right\} \leq g\left(u_{k, \mu}\right)$. Hence

$$
-\Delta\left(u_{\mu}-u_{k, \mu}\right)+\left(g_{k}\left(u_{\mu}\right)-g_{k}\left(u_{k, \mu}\right)\right) \sigma \leq 0 .
$$

Then $u_{\mu} \leq u_{k, \mu}$ by Lemma 3.6. Similarly $u_{k^{\prime}, \mu} \leq u_{k, \mu}$ for $k^{\prime} \geq k>m$. Using $\eta_{1}$ as test-function we obtain

$$
\int_{\Omega}\left(u_{k, \mu}-u_{\mu}\right) d x+\int_{\Omega}\left(g_{k}\left(u_{k, \mu}\right)-g_{k}\left(u_{\mu}\right)\right) \eta_{1} d \sigma=\int_{\Omega}\left(g\left(u_{\mu}\right)-g_{k}\left(u_{\mu}\right)\right) \eta_{1} d \sigma .
$$

Since $g_{k}(r) \rightarrow g(r)$ for any $r \in \mathbb{R}$ and $\left|g_{k}\left(u_{\mu}\right)\right| \leq\left|g\left(u_{\mu}\right)\right|$ with $\rho\left|g\left(u_{\mu}\right)\right| \in L_{\sigma}^{1}(\Omega)$, the right-hand side converges to 0 as $k \rightarrow \infty$ and the second term on the left-hand side is nonnegative. Hence $u_{k, \mu} \rightarrow u_{\mu}$ in $L^{1}(\Omega)$ as $k \rightarrow \infty$, thus $\rho\left(g_{k}\left(u_{k, \mu}\right)-g_{k}\left(u_{\mu}\right)\right) \rightarrow 0$ in $L_{\sigma}^{1}(\Omega)$ which in turn yields $\rho g_{k}\left(u_{k, \mu}\right) \rightarrow \rho g\left(u_{\mu}\right)$ in $L_{\sigma}^{1}(\Omega)$. 
Step 2: proof of I. Denote by $u_{k, \nu}$ the solution of

$$
\begin{array}{rlrl}
-\Delta u+g_{k}(u) & =\nu & & \text { in } \Omega \\
u=0 & & \text { in } \partial \Omega .
\end{array}
$$

Then $-m \leq u_{k, \nu} \leq u_{k, \mu}, u_{k^{\prime}, \mu} \leq u_{k, \mu}$ for $k^{\prime} \geq k>m$ by Lemma 3.6 and $g_{k}\left(u_{k, \nu}\right) \leq$ $g_{k}\left(u_{k, \mu}\right)$. Furthermore $\left\{u_{k, \nu}\right\}$ is bounded in $W_{0}^{1, q}(\Omega)$ for $1<q<\frac{N}{N-1}$ and thus relatively compact in $L^{1}(\Omega)$. Therefore there exists $u \in W_{0}^{1, q}(\Omega)$ such that $u_{k, \nu} \downarrow u$ in $L^{1}(\Omega)$ and also pointwise up to a set with zero $c_{1, q^{-}}$capacity which is therefore a $\sigma$-negligible set. By Step 1, the set $\left\{\rho g_{k}\left(u_{k, \nu}\right)\right\}$ is uniformly integrable in $L_{\sigma}^{1}(\Omega)$, this implies that $u=u_{\nu}$.

Step 3: Proof of II. Because $-\mu^{\prime} \leq \nu \leq \mu$ there holds $u_{k,-\mu^{\prime}} \leq u_{k, \nu} \leq u_{k, \mu}$ and $g_{k}\left(u_{k,-\mu^{\prime}}\right) \leq g_{k}\left(u_{k, \nu}\right) \leq g_{k}\left(u_{k, \mu}\right)$. Since the sets $\left\{u_{k,-\mu^{\prime}}\right\},\left\{u_{k, \nu}\right\}$ and $\left\{u_{k, \mu}\right\}$ are relatively compact in $L^{1}(\Omega)$ and bounded in $W_{0}^{1, q}(\Omega)$ for $1<q<\frac{N}{N-1}$ and the sets $\left\{g_{k}\left(u_{k,-\mu^{\prime}}\right)\right\}$ and $\left\{g_{k}\left(u_{k, \mu}\right)\right\}$ are uniformly integrable in $L_{\sigma}^{1}(\Omega)$, then, up to a subsequence, $u_{k, \nu} \rightarrow u$ in $L^{1}(\Omega)$ and $\sigma$-almost everywhere as $k \rightarrow \infty$. This implies that $g(u) \in L_{\sigma}^{1}(\Omega)$ and $\rho g_{k}\left(u_{k, \nu}\right) \rightarrow \rho g(u)$ in $L_{\sigma}^{1}(\Omega)$. Hence $u=u_{\nu}$.

The proof of the next result, based upon Zorn's lemma, is a variant of the one of $[9$, Th 4.1] which uses inverse maximum principle [9, Corollary 4.8].

Lemma 5.4 Assume $\sigma$ and $g$ satisfy the assumptions of Lemma 5.1. If $\mu \in \mathfrak{M}_{b}^{+}(\Omega)$ there exists a largest good measure smaller than $\mu$, and it is nonnegative.

Proof. Let $\mathcal{Z}_{\mu}$ be the subset of all bounded nonnegative good measures smaller than $\mu$. Notice first that $\mathcal{Z}_{\mu}$ is non-empty since it contains the regular part $\mu_{r}$ of $\mu$ with respect to the $N$-dimensional Hausdorff measure. We now show that $\mathcal{Z}_{\mu}$ is inductive. Let $\mathcal{C}_{I}:=\left\{\mu_{i}\right\}_{i \in I}$ be a totally ordered subset of $\mathcal{Z}_{\mu}$. For $\zeta \in C_{0}(\bar{\Omega}), \zeta \geq 0$, the set of nonnegative real numbers

$$
\mathcal{C}_{I}(\zeta):=\left\{\int_{\Omega} \zeta d \mu_{i}\right\}
$$

is bounded from above by $\int_{\Omega} \zeta d \mu$. Note that can we extend $\mu$ as a positive linear form on $C_{0}(\bar{\Omega})$ since it is a Radon measure and $\mu(\partial \Omega)=0$. Hence $\mathcal{C}_{I}(\zeta)$ admits an upper bound $L(\zeta)$ and there exists a sequence $\left\{i_{k}\right\} \subset I$ such that

$$
\int_{\Omega} \zeta d \mu_{i_{k}} \uparrow L(\zeta) \leq \int_{\Omega} \zeta d \mu \quad \text { as } k \rightarrow \infty
$$

By the Stone-Weiertrass theorem there exists a dense subset $\left\{\zeta_{n}\right\}$ of the set of nonnegative elements in $C_{0}(\bar{\Omega})$. By Cantor diagonal process there exists a subsequence 
$\left\{i_{n_{k}}\right\} \subset I$ such that

$$
\int_{\Omega} \zeta_{n} d \mu_{i_{n_{k}}} \uparrow L\left(\zeta_{n}\right) \leq \int_{\Omega} \zeta_{n} d \mu \quad \text { as } k \rightarrow \infty .
$$

Clearly the map $\zeta_{n} \mapsto L\left(\zeta_{n}\right)$ is additive, positively homogeneous of order one and satisfies

$$
L(\zeta) \leq \int_{\Omega} \zeta d \mu \quad \text { for all } \zeta \in C_{0}(\bar{\Omega}), \zeta \geq 0 .
$$

Hence $L$ extends as a positive linear functional on $C_{0}(\bar{\Omega})$, dominated by $\mu$ denoted by $\mu_{\mathcal{C}_{I}}$. Since $\mu$ is a Radon measure in $\Omega, \mu_{\mathcal{C}_{I}}(\partial \Omega)=0$, hence it is a Radon measure. Furthermore it is a good measure by Lemma 5.1. It follows that $\mu_{\mathcal{C}_{I}} \in \mathcal{Z}_{\mu}$ . Moreover since $L(\zeta)$ is an upper bound of $\mathcal{C}_{I}(\zeta)$ for any nonnegative $\zeta \in C_{0}(\bar{\Omega})$, we have $\mu_{\mathcal{C}_{I}} \geq \mu_{i}$ for any $i \in I$. Hence the set $\mathcal{Z}_{\mu}$ is inductive.

As a consequence of Zorn's lemma, $\mathcal{Z}_{\mu}$ admits at least one maximal element that we denote $\mu^{*}$. If $\nu$ is any nonnegative good measure smaller than $\mu$ it belongs to $\mathcal{Z}_{\mu}$ and hence it cannot dominate $\mu^{*}$. It remains to prove that $\nu \leq \mu^{*}$. Set $\lambda=\sup \left\{\nu, \mu^{*}\right\}$ and let $\lambda^{*}$ be a maximal element of $\mathcal{Z}_{\lambda}$. Since $\nu$ and $\mu^{*}$ are good measures, we have $\nu^{*}=\nu$ and $\left(\mu^{*}\right)^{*}=\mu^{*}$. It follows that $\lambda^{*} \geq \nu^{*}=\nu$ and $\lambda^{*} \geq\left(\mu^{*}\right)^{*}=\mu^{*}$ so that $\lambda^{*} \geq \sup \left\{\nu, \mu^{*}\right\}=\lambda$. This implies that $\lambda^{*}=\lambda \geq \mu^{*}$. On the other hand, since $\nu, \mu^{*} \leq \mu$, we have $\lambda \leq \mu$ and thus $\lambda^{*} \leq \mu$. By definition of a maximal element it implies that $\lambda^{*}=\lambda=\mu^{*}$, and finally $\mu^{*}=\sup \left\{\nu, \mu^{*}\right\}$. We infer $\nu \leq \mu^{*}$ and then $\mu^{*}$ is the maximum of $\mathcal{Z}_{\mu}$.

Corollary 5.5 Assume $\sigma$ and $g$ satisfy the assumptions of Lemma 5.1. If $\mu, \nu \in$ $\mathfrak{M}_{b}^{+}(\Omega)$ are good measures, then $\sup \{\mu, \nu\}$ is a good measure.

Proof. Set $\lambda=\sup \{\mu, \nu\}$. Then

$$
\lambda \geq \lambda^{*}=(\sup \{\mu, \nu\})^{*} \geq \sup \left\{\mu^{*}, \nu^{*}\right\}=\sup \{\mu, \nu\}=\lambda .
$$

This implies $\lambda=\lambda^{*}$, hence $\lambda$ is a good measure.

\subsection{The capacitary framework}

We start with the following regularity estimate for the Poisson problem

Lemma 5.6 For any $s \geq 0$ and $1<p<\infty$, the mapping $\mu \mapsto \mathbb{G}[\mu]$ is continuous from $\mathfrak{M}_{b}(\Omega) \cap H^{s-2, p}(\Omega)$ to $H^{s, p}(\Omega)$.

Proof. It is classical that the mapping $G_{D}: \lambda \mapsto u=G_{D}(\lambda)$ solution of $-\Delta u=\lambda$ in $\Omega$ and $u=0$ on $\partial \Omega$ is continuous from $H^{s-2, p}(\Omega)$ to $H^{s, p}(\Omega)$ for $1<p<\infty$ 
and $s>\frac{1}{p}$ (see e.g. [14, Example 3.15 p. 314]). Thus we are left with the case $0 \leq s \leq \frac{1}{p}$. If $\lambda \in \mathfrak{M}_{b}(\Omega)$, then $G_{D}(\lambda)=\mathbb{G}[\lambda]$ is a very weak solution, hence, since $\begin{aligned} \mathbb{X}(\Omega) \subset & C_{c}^{1}(\bar{\Omega}) \cap\left(\bigcap_{1<r<\infty} H^{2, r}(\Omega)\right), \\ & -\int_{\Omega} G_{D}(\lambda) \Delta \zeta d x=\int_{\Omega} \zeta d \lambda \leq\|\zeta\|_{H^{2-s, p^{\prime}}}\|\lambda\|_{H^{s-2, p}} \quad \text { for all } \zeta \in \mathbb{X}(\Omega) .\end{aligned}$

In particular, if $\zeta=\mathbb{G}[v]$, then $\|\zeta\|_{H^{2-s, p^{\prime}}} \leq c\|v\|_{H^{-s, p^{\prime}}}$ since $-s>-2+1 / p^{\prime}$, and

$$
\int_{\Omega} G_{D}(\lambda) v d x \leq c\|v\|_{H^{-s, p^{\prime}}}\|\lambda\|_{H^{s-2, p}} \quad \text { for all } v \in \Delta(\mathbb{X}(\Omega)) .
$$

In particular this inequality holds if $v \in C_{c}(\bar{\Omega})$ which is dense in $H^{-s, p^{\prime}}(\Omega)$. Finally this inequality means that the mapping $v \mapsto \int_{\Omega} G_{D}(\lambda) v d x$ is a continuous linear form over $H^{-s, p^{\prime}}(\Omega)$, it thus belongs to $H^{s, p}(\Omega)$.

Proposition 5.7 Let $\sigma$ and $g$ satisfy the assumptions in Theorem E. If $\mu \in \mathfrak{M}_{b}(\Omega)$ is such that $|\mu| \in H^{s-2, p}(\Omega)$ for some $p>1$ and $s>0$ such that $N-\theta<s p<N$ and $\frac{\theta p}{N-s p} \geq q$, then (1.3) admits a unique very weak solution.

Proof. By Lemma 5.6, if $|\mu| \in H^{s-2, p}(\Omega)$ then $\mathbb{G}[|\mu|] \in H^{s, p}(\Omega)$. By Proposition 2.4

$$
\|\mathbb{G}[|\mu|]\|_{L_{\sigma}^{q}} \leq c\|\mathbb{G}[|\mu|]\|_{H^{s, p}}
$$

if and only if $\sigma \in \mathcal{M}_{r}^{+}(\Omega)$ with $\frac{1}{r}=q\left(\frac{1}{q}-\frac{1}{p}+\frac{s}{N}\right)=\frac{N-\theta^{\prime}}{N}$. Then $q=\frac{\theta^{\prime} p}{N-s p}$. Hence, if $\frac{\theta p}{N-s p} \geq q$ we get $\theta \geq \theta^{\prime}$ and then $\mathcal{M}_{\frac{N}{N-\theta}}^{+}(\Omega) \subset \mathcal{M}_{\frac{N}{N-\theta^{\prime}}}^{+}(\Omega)$ by [2.7). We conclude by Theorem D.

Remark. This result covers the case $q=p$, in which any bounded measure such that $|\mu| \in H^{\frac{N-\theta}{q}-2, q}\left(\mathbb{R}^{N}\right)$ is eligible for solving problem (1.2).

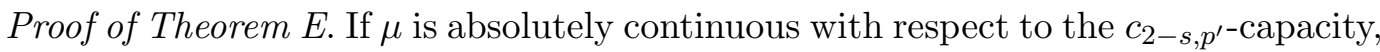
so are $\mu^{+}$and $-\mu^{-}$. By [13] there exists an increasing sequence of positive bounded Radon measures $\mu_{j} \in H^{s-2, p}(\Omega)$ converging to $\mu^{+}$. By Proposition $5.7 \mu_{j}$ is a good measure, hence by Lemma $5.1 \mu^{+}$is a good measure. In the same way $-\mu^{-}$is a good measure. Since $-\mu_{-} \leq \mu \leq \mu_{+}$, it follows from Lemma 5.3-II that $\mu$ is a good measure.

Proof of Proposition 1.1. Notice first that if $\mu \in \mathcal{M}_{\frac{N}{N-\theta^{*}}}(\Omega)$ with $\theta^{*}>N-s p$, then for any compact $K \subset \Omega$,

$$
|\mu|(K) \leq c^{\prime}\left(c_{s, p}(K)\right)^{\frac{1}{p}}
$$


In particular $\mu$ is absolutely continuous w.r.t $c_{s, p}$-capacity. Indeed under the assumption on $\theta^{*}$ we have $H^{s, p}(\Omega) \hookrightarrow L_{|\mu|}^{1}(\Omega)$. It follows that for any $v \in H^{s, p}(\Omega)$, $v \geq 1$ on $K$, we have

$$
|\mu|(K) \leq \int_{K} v d|\mu| \leq\|v\|_{L_{|\mu|}^{1}} \leq C\|v\|_{H^{s, p}}
$$

We deduce (5.15) taking the infimum over $v$. To apply Theorem $\mathrm{E}$ we need $\mu$ to be $c_{2-\frac{N-\theta}{q}, q^{\prime}}$-diffuse. It thus suffices to take $\theta^{*}>N-s p$ with $s=2-\frac{N-\theta}{q}$ and $p=q^{\prime}$. We obtain exactly the condition on $\theta^{*}$ stated in Proposition 1.1.

\subsection{The case $g(u)=|u|^{q-1} u$.}

In the sequel we consider the following equation

$$
\begin{aligned}
-\Delta u+|u|^{q-1} u \sigma & =\mu & & \text { in } \Omega \\
u & =0 & & \text { in } \partial \Omega,
\end{aligned}
$$

where $q>1$. A measure for which there exists a solution, necessarily unique by Lemma 3.5, is called q-good. Assume that $\sigma \in \mathcal{M}_{\frac{N}{N-\theta}}^{+}$with $N \geq \theta>N-\frac{N}{N-1}$. Then the critical exponent $q$ from the point of view of (1.8) in Theorem B is

$$
q_{\theta}:=\frac{\theta}{N-2}
$$

which is larger than 1 if $N>2$.

Let $q>1$ and $\sigma \in \mathfrak{M}_{b}^{+}(\Omega)$. Recall that the Green function $G$ of the Dirichlet Laplacian in $\Omega$ is defined on $\bar{\Omega} \times \bar{\Omega}$ with values in $[0,+\infty]$ with $G(x, x)=+\infty$, $x \in \Omega$, and $G(x, y)=0$ if $x \in \partial \Omega$ or $y \in \partial \Omega$. We extend $G$ to $\mathbb{R}^{N} \times \bar{\Omega}$ by setting $G(x, y)=0$ if $(x, y) \in \bar{\Omega}^{c} \times \bar{\Omega}$. Hence $x \mapsto G(x, y)$ is lower semicontinuous in $\mathbb{R}^{N}$ and $y \mapsto G(x, y)$ is lower semicontinuous in $\Omega$, and thus is $\sigma$-measurable. Following [2, Sec. 2.3] we then consider the following set function with values in $[0,+\infty]$,

$$
c_{q}^{\sigma}(E)=\inf \left\{\int_{\Omega}|v|^{q^{\prime}} d \sigma: v \in L_{\sigma}^{q^{\prime}}(\Omega), \mathbb{G}[v \sigma](x) \geq 1 \text { for all } x \in E\right\},
$$

for any $E \subset \Omega$. According to the general theory developped in [2, Sec. 2.3] $c_{q}^{\sigma}$ is a regular capacity in the sense of Choquet. Using the lower semicontinuity of $y \mapsto \mathbb{G}[v \sigma](y)$ (see [2, Prop 2.3.2]) it is easy to verify that for any compact set $K \subset \Omega$, there holds

$$
c_{q}^{\sigma}(K)=\inf \left\{\int_{\Omega}|v|^{q^{\prime}} d \sigma: v \in L_{\sigma}^{\infty}(\Omega), \mathbb{G}[v \sigma](x) \geq 1 \text { for all } x \in K\right\} .
$$


The dual formulation of the capacity is the following (see [2, Th 2.5.1]),

$$
\left(c_{q}^{\sigma}(K)\right)^{\frac{1}{q^{\prime}}}=\sup \left\{\lambda(K): \lambda \in \mathfrak{M}_{b}^{+}(K),\|\mathbb{G}[\lambda]\|_{L_{\sigma}^{q}} \leq 1\right\}
$$

for $K \subset \Omega, K$ compact. Existence of extremal measures satisfying equality in (5.20) is proved in [2, Th 2.5.3].

Remark. Note that the $\geq$ inequality in (5.20) follows directly from the following one

$$
\nu(K) \leq\left(c_{q}^{\sigma}(K)\right)^{\frac{1}{q^{\prime}}}\|\mathbb{G} \nu\|_{L_{\sigma}^{q}}
$$

which holds for any $\nu \in \mathfrak{M}_{b}^{+}(\Omega)$ such that $\mathbb{G}[\nu] \in L_{\sigma}^{q}$ and any $K \subset \Omega$ compact.

We now give some sufficient conditions for a bounded measure to be absolutely continuous with respect to the capacity $c_{q}^{\sigma}$. First in view of (5.21) and the dual expression of the capacity it is clear that there holds:

Lemma 5.8 If $\nu \in \mathfrak{M}_{b}(\Omega)$ is such that $\mathbb{G}[|\nu|] \in L_{\sigma}^{q}(\Omega)$, then $\nu$ is absolutely continuous with respect to the capacity $c_{q}^{\sigma}$. This holds in particular if $\nu \in \mathfrak{M}_{b}(\Omega)$ is such that $|\nu| \in H^{s-2, p}(\Omega)$ for some $p>1$ and $s>0$ verifying $N-\theta<s p<N$ and $\frac{\theta p}{N-s p} \geq q$.

As a direct consequence we have

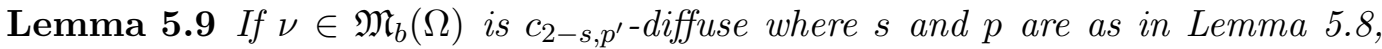
then $\nu$ is absolutely continuous with respect to the capacity $c_{q}^{\sigma}$.

Proof. If $\nu \geq 0$ there exists a sequence of nonnegative measures $\left\{\nu_{n}\right\} \subset H^{s-2, p}(\Omega)$ such that $\nu_{n} \uparrow \nu$. If $K$ is a compact such that $c_{q}^{\sigma}(K)=0$ then $\nu_{n}(K)=0$ by Lemma 5.8 and thus $\nu(K)=0$. When $\nu$ is a signed measure, we apply the above to its positive and negative part $\nu^{ \pm}$.

The following particular case will be useful:

Lemma 5.10 If $\nu \in \mathcal{M}_{\frac{N}{N-\theta}}(\Omega)$ with $N \geq \theta>N-2$, then $\nu$ is absolutely continuous with respect to the capacity $c_{q}^{\sigma}$.

Proof. We have $|\nu| \in \mathcal{M}_{p}(\Omega)$ for some $p>\frac{N}{2}$. We then obtain from (2.9) that $\mathbb{G}[|\nu|]$ is bounded so that $\mathbb{G}[|\nu|] \in L_{\sigma}^{q}(\Omega)$. The conclusion follows from the previous lemma.

Remark. It is noticeable that if the support of a nonnegative measure $\mu$ does not intersect the support of $\sigma$, then $\mu$ is always $q$-good. This is due to the fact that $\mathbb{G}[\mu]$ is bounded on the support of $\sigma$, hence $\mathbb{G}[\mu] \in L_{\sigma}^{q}(\Omega)$ for any $q<\infty$ and the result 
follows from Theorem D. Hence, a more accurate necessary condition must involve a notion of density of $\sigma$ on its support, a property which has been developed by Triebel [26] in connection with fractal measures.

We recall that the $\theta$-dimensional Hausdorff measure $H^{\theta}, 0 \leq \theta \leq N$, is defined on subsets $E$ of $\mathbb{R}^{N}$ by

$$
H^{\theta}(E)=\lim _{\delta \rightarrow 0}\left(\inf \left\{\sum_{j=1}^{\infty}\left(\operatorname{diam} U_{j}\right)^{\theta}: E \subset \bigcup_{j=1}^{\infty} U_{j}, \operatorname{diam} U_{j} \leq \delta\right\}\right) .
$$

Definition 5.11 A nonnegative Radon measure $\sigma$ on $\bar{\Omega}$ with support $\Gamma$ is $\theta$-regular with $0 \leq \theta \leq N$ if there exists $c>0$ such that

$$
\frac{1}{c} r^{\theta} \leq\left|B_{r}(x)\right|_{\sigma} \leq c r^{\theta} \quad \text { for all } x \in \Gamma \text {, for all } r>0 .
$$

The support $\Gamma$ of $\sigma$ is called a $\theta$-set.

By [26, Th 3.4] $\sigma$ is equivalent in $\bar{\Omega}$ to the restriction $H^{\theta}\left\lfloor_{\Gamma}\right.$ of $H^{\theta}$ to $\Gamma$ in the sense that there exists $c^{\prime}>0$ such that

$$
\frac{1}{c^{\prime}} H^{\theta}(E \cap \Gamma) \leq \sigma(E) \leq c^{\prime} H^{\theta}(E \cap \Gamma) \quad \text { for all } E \subset \bar{\Omega}, E \text { Borel. }
$$

The description of $L_{\sigma}^{p}(\Gamma)$ necessitates to introduce the scale of Besov spaces and their trace on $\Gamma$. For $0<s<1,1 \leq p, q \leq \infty$, we denote by $B_{p, q}^{s}(\Omega)$ the space obtained by the real interpolation method by

$$
B_{p, q}^{s}(\Omega)=\left[W^{1, p}(\Omega), L^{p}(\Omega)\right]_{s, q} .
$$

Details can be found in [23]. Its norm is equivalent to

$$
\|\phi\|_{B_{p, q}^{s}}=\|v\|_{L^{p}}+\left(\int_{0}^{\infty} \frac{\left(\omega_{p}(t ; v)\right)^{q}}{t^{s q}} \frac{d t}{t}\right)^{\frac{1}{q}}
$$

if $q<\infty$ and

$$
\|\phi\|_{B_{p, \infty}^{s}}=\|v\|_{L^{p}}+\sup _{t>0} \frac{\omega_{p}(t ; v)}{t^{s}}
$$

where

$$
\omega_{p}(t ; \phi)=\sup _{|h|<t}\|v(.+h)-v(.)\|_{L^{p}} .
$$

For $k \in \mathbb{N}_{*}, B_{p, q}^{k+s}(\Omega)=\left\{v \in W^{k, p}(\Omega): D^{\alpha} v \in B_{p, q}^{s}(\Omega)\right.$, for all $\left.\alpha \in \mathbb{N}^{N},|\alpha|=k\right\}$ with norm

$$
\|v\|_{B_{p, q}^{k+s}}=\|v\|_{W^{k-1, p}}+\sum_{|\alpha|=k}\left\|D^{\alpha} v\right\|_{B_{p, q}^{s}} .
$$


If $\Gamma \subset \mathbb{R}^{N}$ is a closed set with zero Lebesgue measure, we consider the set

$$
B_{p, q}^{s, \Gamma}\left(\mathbb{R}^{N}\right)=\left\{v \in B_{p, q}^{s}\left(\mathbb{R}^{N}\right):\langle v, \phi\rangle=0 \quad \text { for all } \phi \in \mathcal{S}\left(\mathbb{R}^{N}\right) \text { s.t. } \phi\left\lfloor_{\Gamma}=0\right\},\right.
$$

endowed with the $B_{p, q}^{s}\left(\mathbb{R}^{N}\right)$ norm, where $\langle v, \phi\rangle$ is the pairing between $\mathcal{S}^{\prime}\left(\mathbb{R}^{N}\right)$ and $\mathcal{S}\left(\mathbb{R}^{N}\right)$. If $v \in L_{\sigma}^{p}(\Omega)$ and $\sigma$ has support $\Gamma \subset \bar{\Omega}$, the linear map

$$
\phi \mapsto T_{v}^{\sigma}(\phi)=\int_{\Gamma} \phi v d \sigma
$$

defined on $\mathcal{S}\left(\mathbb{R}^{N}\right)$ is a tempered distribution in $\mathbb{R}^{N}$. The following results are proved in $[26$, Th 18.2, 18.6].

Proposition $\mathbf{5 . 1 2}$ Assume $\sigma$ is $\theta$-regular, $0<\theta<N$, with support $\Gamma \subset \mathbb{R}^{N}$, and let $v \in L_{\sigma}^{q}(\Omega)$ with $1<p \leq+\infty$. There holds

$$
\left|T_{v}^{\sigma}(\phi)\right| \leq c\|v\|_{L_{\sigma}^{p}}\|\phi\|_{B_{p^{\prime}, 1}^{\frac{N-\theta}{p^{\prime}}}} \quad \text { for all } \phi \in \mathcal{S}\left(\mathbb{R}^{N}\right) .
$$

It follows that $T_{v}^{\sigma} \in B_{p, \infty^{-\frac{N-\theta}{p^{\prime}}}, \Gamma}$ with $\left\|T_{v}^{\sigma}\right\|_{\substack{B_{p, \infty} \\-\frac{N-\theta}{p^{\prime}}}} \leq c\|v\|_{L_{\sigma}^{p}}$.

Morover the map $v \in L_{\sigma}^{p}(\Gamma) \rightarrow T_{v}^{\sigma} \in B_{p, \infty^{-\frac{N-\theta}{p^{\prime}}}, \Gamma}$ is a linear isomorphism. We can thus denote $L_{\sigma}^{p}(\Gamma) \sim\left(B_{p^{\prime}, 1}^{\frac{N-\theta}{p^{\prime}}, \Gamma}\right)^{\prime}=B_{p, \infty}^{-\frac{N-\theta}{p^{\prime}}, \Gamma}$.

Proposition 5.13 Assume $\sigma$ is $\theta$-regular, $0<\theta<N$ with support $\Gamma \subset \mathbb{R}^{N}$. Then for any $1<p \leq \infty$ the restriction operation from $\mathcal{S}\left(\mathbb{R}^{N}\right)$ to $C(\Gamma), \phi \mapsto \phi\left\lfloor_{\Gamma}\right.$ can be extended as a continuous linear operator from $B_{p, 1}^{\frac{N-\theta}{p}}\left(\mathbb{R}^{N}\right)$ to $L_{\sigma}^{p}(\Gamma)$ that we denote $\operatorname{Tr}_{\Gamma}$. Furthermore this operator is onto.

Definition 5.14 If $\sigma \in \mathfrak{M}_{b}^{+}(\Omega)$ is $\theta$-regular, $N \geq \theta>N-2$ with support $\Gamma \subset \Omega$ and $m, q>1$, we set

$$
\left.c_{q^{\prime}, \infty}^{2-\frac{N-\theta}{q}, \Gamma} K\right)=\inf \left\{\|\zeta\|_{B_{q^{\prime}, \infty}^{2-\frac{N-\theta}{q}}}^{q^{\prime}}: \zeta \in B_{q^{\prime}, \infty}^{2-\frac{N-\theta}{q}, \Gamma}(\Omega) \text { s.t. } \zeta \geq \chi_{K}\right\}
$$

where

$$
B_{q^{\prime}, \infty}^{2-\frac{N-\theta}{q}, \Gamma}(\Omega)=\left\{\zeta \in B_{q^{\prime}, \infty}^{2-\frac{N-\theta}{q}}(\Omega) \text { s.t. } \Delta \zeta \in B_{q^{\prime}, \infty}^{-\frac{N-\theta}{q}, \Gamma}(\Omega)\right\} .
$$

Notice that $B_{q^{\prime}, \infty}^{2-\frac{N-\theta}{q}, \Gamma}(\Omega)$ is a closed subspace of $B_{q^{\prime}, \infty}^{2-\frac{N-\theta}{q}}(\Omega)$. 
Proposition 5.15 Assume $\sigma \in \mathfrak{M}_{b}^{+}(\Omega)$ is $\theta$-regular, $N \geq \theta>N-2$ with support $\Gamma \subset \Omega$ and $q>1$. Then there exists a positive constant $M>0$ such that

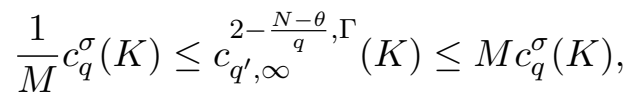

for all compact set $K \subset \Omega$.

Proof. By standard elliptic equations and interpolation theory (see [23], [24]), for any $\psi \in B_{q^{\prime}, \infty}^{-\frac{N-\theta}{q}, \Gamma}(\Omega), \mathbb{G}[\psi \sigma] \in B_{q^{\prime}, \infty}^{2-\frac{N-\theta}{q}}(\Omega)$ and there holds

$$
\frac{1}{c}\|\mathbb{G}[\psi \sigma]\|{ }_{B_{q^{\prime}, \infty}^{2-\frac{N-\theta}{q}}} \leq\|\psi\|_{B_{q^{\prime}, \infty}^{-\frac{N-\theta}{q}, \Gamma}} \leq c\|\mathbb{G}[\psi \sigma]\|_{B_{q^{\prime}, \infty}^{2-\frac{N-\theta}{q}}} .
$$

By Proposition 5.12 we can replace $\|\psi\|_{B_{q^{\prime}, \infty}^{-\frac{N-\theta}{q}, \Gamma}}$ by $\|\psi\|_{L_{\sigma}^{q^{\prime}}}$ in the above inequality, up to a change of constants $c$. Let $\left\{v_{k}\right\} \subset L_{\sigma}^{q^{\prime}, \infty}(\Omega)$ be such that $v_{k} \geq 0, \zeta_{k}:=\mathbb{G}\left[v_{k} \sigma\right] \geq 0$ on $K$ and $\left\|v_{k}\right\|_{L_{\sigma}^{q^{\prime}}} \downarrow\left(c_{q}^{\sigma}(K)\right)^{\frac{1}{q^{\prime}}}$. Since (5.32) is equivalent to

$$
\left.\left.\frac{1}{c} \| \zeta_{k}\right]\left\|_{B_{q^{\prime}, \infty}^{2-\frac{N-\theta}{q}}} \leq\right\| v_{k}\left\|_{L_{\sigma}^{q^{\prime}}} \leq c\right\| \zeta_{k}\right] \|_{B_{q^{\prime}, \infty}^{2-\frac{N-\theta}{q}}},
$$

we derive $c_{q^{\prime}, \infty}^{2-\frac{N-\theta}{q}, \Gamma}(K) \geq \frac{1}{c^{q^{\prime}}} c_{q}^{\sigma}(K)$. Similarly $c_{q^{\prime}, \infty}^{2-\frac{N-\theta}{q}, \Gamma}(K) \leq c^{q^{\prime}} c_{q}^{\sigma}(K)$.

Proof of Theorem F. By Lemma 5.10 the measure $u^{q}$ vanishes on Borel sets with zero $c_{q}^{\sigma}$-capacity. Since $u \in L_{\sigma}^{q}(\Omega)$ the mapping

$$
\phi \mapsto \int_{\Gamma} u \phi d \sigma=\langle u, \phi\rangle
$$

is a tempered distribution that we denote by $T_{u}^{\sigma}$, hence

$$
|\langle\Delta u, \phi\rangle|=|\langle u, \Delta \phi\rangle|=\left|\int_{\Omega} u \Delta \phi d \sigma\right| \leq\|u\|_{L_{\sigma}^{q}}\|\Delta \phi\|_{L_{\sigma}^{q^{\prime}}}
$$

Using Proposition 5.12

$$
\|\Delta \phi\|_{L_{\sigma}^{q^{\prime}}} \leq c\|\Delta \phi\|_{B_{q^{\prime}, \infty}^{-\frac{N-\theta}{q}, \Gamma}} \leq c^{\prime}\|\phi\|_{B_{q^{\prime}, \infty}^{2-\frac{N-\theta}{q}, \Gamma}} .
$$

Therefore the nonnegative measure $T_{u}^{\sigma}$ is a continuous linear form on $B_{q^{\prime}, \infty}^{2-\frac{N-\theta}{q}, \Gamma}(\Omega)$. Therefore it vanishes on Borel sets with zero $c_{q^{\prime}, \infty}^{2-\frac{N-\theta}{q}, \Gamma}$-capacity, which actually coincide with Borel sets with zero zero $c_{q}^{\sigma}$-capacity. 


\subsection{Removable singularities}

It is easy to prove that for any compact set $K \subset \Omega$, there exists $\mu_{K} \in \mathfrak{M}_{b}^{+}(K)$ such that $\int_{\Omega}\left(\mathbb{G}\left[\mu_{K}\right]\right)^{q} d \sigma=1$ and $c_{q}^{\sigma}(K)=\mu_{K}(K)$ (see [2][Th 2.5.3]). Since $\mu_{K}$ is an admissible measure, it follows from Theorem D that (1.3) is solvable with $\mu=\mu_{K}$, hence $K$ is not removable. Although it could be conjectured that a compact set with zero $c_{q}^{\sigma}$-capacity is removable we can prove this assertion only for sigma-moderate solutions.

Definition 5.16 Let $q>1, \sigma \in \mathcal{M}_{\frac{N}{N-\theta}}^{+}(\Omega)$ where $N \geq \theta>N-2$ and $K \subset \Omega$ a compact set. A nonnegative function $u \in L_{l o c}^{1}(\bar{\Omega} \backslash K) \cap L_{\sigma, l o c}^{q}(\bar{\Omega} \backslash K)$ is a sigmamoderate solution of

$$
\begin{aligned}
-\Delta u+|u|^{q-1} u \sigma & =0 & & \text { in } \Omega \backslash K \\
u & =0 & & \text { in } \partial \Omega,
\end{aligned}
$$

if there exists an increasing sequence $\left\{\mu_{n}\right\} \subset \mathfrak{M}_{b}^{+}(K)$ of q-good measures such that $u_{\mu_{n}} \rightarrow u$ in $L_{l o c}^{1}(\bar{\Omega} \backslash K) \cap L_{\sigma, l o c}^{q}(\bar{\Omega} \backslash K)$.

Theorem 5.17 Under the assumptions on $q, \sigma$ and $K$ of Definition 5.16, if $c_{q}^{\sigma}(K)=$ 0 then the only sigma-moderate solution of (5.35) is the trivial one.

Proof. Since $c_{q}^{\sigma}(K)=0$ the set of nonnegative $q$-good measures with support in $K$ is reduced to the zero function by Theorem F. This implies the claim.

Remark. We conjecture that for any compact set $K \subset \Omega$, any nonnegative local solution of (5.12) is sigma-moderate. This would imply that a necessary and sufficient condition for a local nonnegative solution of (5.12) to be a solution in $\Omega$ is $c_{q}^{\sigma}(K)=0$. However this type of result is usually difficult to prove, see [22], [17], [12] in the framework of semilinear equations with measure boundary data.

In order to find necessary and sufficient conditions for the removability of a compact set $K \subset \Omega$, we assume that $\sigma$ is a positive measure in $\Omega$ absolutely continuous with respect to the Lebesgue measure, with a nonnegative density $w$. For proving our results we will assume that the function $\omega=w^{-\frac{1}{q-1}}$ is $q^{\prime}$-admissible in the sense of [15, Chap 1]. One sufficient condition is that $w$ belongs to the Muckenhoupt class $A_{q}$, that is

$$
\sup _{B}\left(\frac{1}{|B|} \int_{B} w d x\right)\left(\frac{1}{|B|} \int_{B} w^{-\frac{1}{q-1}} d x\right)^{\frac{1}{p-1}}=m_{w, q}<\infty
$$


for all ball $B \subset \mathbb{R}^{N}$.

If $K \subset \Omega$ is compact, we set

$$
c_{q}^{\omega}(K)=\inf \left\{\int_{\Omega}|\Delta \zeta|^{q^{\prime}} \omega d x: \zeta \in C_{0}^{\infty}(\Omega), \zeta \geq 1 \text { in a neighborhood of } K\right\} .
$$

This defines a capacity on Borel subsets of $\Omega$. Since $\omega$ is $q^{\prime}$-admissible, it satisfies Poincaré inequality, hence a set with zero $c_{q}^{\omega}$-capacity is $\omega$-negligible. Furthermore, following the proof of $\left[2\right.$, Th 3.3.3], $c_{q}^{\omega}$ is equivalent to $\dot{c}_{q}^{\omega}$ defined by

$$
\dot{c}_{q}^{\omega}(K)=\inf \left\{\|\zeta\|_{W_{\omega}^{2, q^{\prime}}}^{q^{\prime}}: \zeta \in C_{0}^{\infty}(\Omega), 0 \leq \zeta \leq 1, \zeta \geq 1 \text { in a neighborhood of } K\right\} .
$$

The dual definition is (see [2, Th 2.5.1])

$$
\left(c_{q}^{\omega}(K)\right)^{\frac{1}{q^{\prime}}}=\sup \left\{\lambda(K): \lambda \in \mathfrak{M}_{b}^{+}(K),\|\mathbb{G}[\lambda]\|_{L_{\omega}^{q}} \leq 1\right\} .
$$

Proof of Theorem G. Step 1: The condition is sufficient. We assume first that $L_{w, l o c}^{q}(\Omega \backslash K) \cap u \in L^{1}(\Omega \backslash K)$ is a nonnegative subsolution of (1.22) in the sense of distributions in $\Omega \backslash K$ where $K \subset \Omega$ is a compact subset with $c_{q}^{\omega}$-capacity zero. There exists a sequence of functions $\left\{\zeta_{k}\right\} \subset C_{0}^{\infty}(\Omega)$ with value in $[0,1]$, value 1 in a neighborhood of $K$ and such that $\left\|\Delta \zeta_{k}\right\|_{L_{\omega}^{q^{\prime}}} \rightarrow 0$ when $k \rightarrow \infty$. Let $\rho \in C_{0}^{\infty}(\Omega)$, $0 \leq \rho \leq 1$, such that $\rho=1$ in a neighborhood of $K$ containing the support of the $\zeta_{k}$. Using $\phi_{k}:=\left(1-\zeta_{k}\right)^{\alpha} \rho^{\alpha}$, with $\alpha>1$, in the very weak formulation of equation (1.22) we obtain,

$$
\begin{aligned}
\int_{\Omega} u^{q} \phi_{k} w d x & \leq \int_{\Omega} u \Delta \phi_{k} d x \\
& \leq \alpha \int_{\Omega} u\left(1-\zeta_{k}\right)^{\alpha} \rho^{\alpha-1} \Delta \rho d x-2 \alpha \int_{\Omega} u\left(1-\zeta_{k}\right)^{\alpha-1} \nabla \zeta_{k} . \nabla \rho^{\alpha} d x \\
& -\alpha \int_{\Omega} u\left(1-\zeta_{k}\right)^{\alpha-1} \rho^{\alpha} \Delta \zeta_{k} d x+\alpha(\alpha-1) \int_{\Omega} u\left(1-\zeta_{k}\right)^{\alpha-2} \rho^{\alpha}\left|\nabla \zeta_{k}\right|^{2} d x \\
& +\alpha(\alpha-1) \int_{\Omega} u\left(1-\zeta_{k}\right)^{\alpha} \rho^{\alpha-2}|\nabla \rho|^{2} d x .
\end{aligned}
$$

Notice that the second integral in the right-hand side vanishes since $\nabla \zeta_{k} . \nabla \rho^{\alpha}=0$ by the assumption on their support. If we choose $\alpha=2 q^{\prime}$, we can bound the remaining 
integrals as follows:

$$
\begin{aligned}
&\left|\int_{\Omega} u\left(1-\zeta_{k}\right)^{2 q^{\prime}-1} \rho^{2 q^{\prime}} \Delta \zeta_{k} d x\right| \leq\left(\int_{\Omega} u^{q} \phi_{k} w d x\right)^{\frac{1}{q}}\left(\int_{\Omega}\left|\Delta \zeta_{k}\right|^{q^{\prime}}\left(1-\zeta_{k}\right)^{q^{\prime}} \rho^{2 q^{\prime}} \omega d x\right)^{\frac{1}{q^{\prime}}} \\
& \leq\left(\int_{\Omega} u^{q} \phi_{k} w d x\right)^{\frac{1}{q}}\left(\int_{\Omega}\left|\Delta \zeta_{k}\right|^{q^{\prime}} \omega d x\right)^{\frac{1}{q^{\prime}}}, \\
&\left|\int_{\Omega} u\left(1-\zeta_{k}\right)^{2 q^{\prime}} \rho^{2 q^{\prime}-1} \Delta \rho d x\right| \leq\left(\int_{\Omega} u^{q} \phi_{k} w d x\right)^{\frac{1}{q}}\left(\int_{\Omega}|\Delta \rho|^{q^{\prime}}\left(1-\zeta_{k}\right)^{2 q^{\prime}} \rho^{q^{\prime}} \omega d x\right)^{\frac{1}{q^{\prime}}} \\
& \leq\left(\int_{\Omega} u^{q} \phi_{k} w d x\right)^{\frac{1}{q}}\left(\int_{\Omega}|\Delta \rho|^{q^{\prime}} \omega d x\right)^{\frac{1}{q^{\prime}}}, \\
&\left.\left|\int_{\Omega} u\left(1-\zeta_{k}\right)^{2 q^{\prime}-2} \rho^{2 q^{\prime}}\right| \nabla \zeta_{k}\right|^{2} d x \mid \leq\left(\int_{\Omega} u^{q} \phi_{k} w d x\right)^{\frac{1}{q}}\left(\int_{\Omega}\left|\nabla \zeta_{k}\right|^{2 q^{\prime}} \rho^{2 q^{\prime}} \omega d x\right)^{\frac{1}{q^{\prime}}} \\
& \leq\left(\int_{\Omega} u^{q} \phi_{k} w d x\right)^{\frac{1}{q}}\left(\int_{\Omega}\left|\nabla \zeta_{k}\right|^{2 q^{\prime}} \omega d x\right)^{\frac{1}{q^{\prime}}},
\end{aligned}
$$

and finally

$$
\begin{aligned}
\left.\left|\int_{\Omega} u\left(1-\zeta_{k}\right)^{2 q^{\prime}} \rho^{2 q^{\prime}-2}\right| \nabla \rho\right|^{2} d x \mid & \leq\left(\int_{\Omega} u^{q} \phi_{k} w d x\right)^{\frac{1}{q}}\left(\int_{\Omega}|\nabla \rho|^{2 q^{\prime}}\left(1-\zeta_{k}\right)^{2 q^{\prime}} \omega d x\right)^{\frac{1}{q^{\prime}}} \\
& \leq\left(\int_{\Omega} u^{q} \phi_{k} w d x\right)^{\frac{1}{q}}\left(\int_{\Omega}|\nabla \rho|^{2 q^{\prime}} \omega d x\right)^{\frac{1}{q^{\prime}}} .
\end{aligned}
$$

Since the Gagliardo-Nirenberg inequality holds with the $q^{\prime}$-admissible weight $\omega$, we have for some $\tau \in(0,1)$ and some $c=c(q, N)>0$,

$$
\begin{aligned}
\left(\int_{\Omega}\left|\nabla \zeta_{k}\right|^{2 q^{\prime}} \omega d x\right)^{\frac{1}{2 q^{\prime}}} & \leq c\left(\int_{\Omega}\left|\Delta \zeta_{k}\right|^{q^{\prime}} \omega d x\right)^{\frac{\tau}{q^{\prime}}}\left\|\zeta_{k}\right\|_{L^{\infty}}^{1-\tau} \\
& \leq c^{\prime}\left(\int_{\Omega}\left|\Delta \zeta_{k}\right|^{q^{\prime}} \omega d x\right)^{\frac{\tau}{q^{\prime}}} .
\end{aligned}
$$

Therefore, if we set

$$
X_{k}=\left(\int_{\Omega} u^{q} \phi_{k} w d x\right)^{\frac{1}{q}} \quad \text { and } \quad Z_{k}=\left(\int_{\Omega}\left|\Delta \zeta_{k}\right|^{q^{\prime}} \omega d x\right)^{\frac{1}{q^{\prime}}}
$$

we obtain the inequation

$$
X_{k}^{q} \leq c_{1} X_{k} Z_{k}+c_{2} X_{k}+c_{3} X_{k} Z_{k}^{\tau}
$$


for some positive constants $c_{1}, c_{2}, c_{3}$ depending on $q, N$ and $\rho$. By definition of $\zeta_{k}$ we have $Z_{k} \rightarrow 0$. We thus deduce that $X_{k}^{q} \leq c X_{k}$ with $q>1$ and then that the sequence $\left\{X_{k}\right\}$ is bounded. Since $\zeta_{k} \rightarrow 0$ almost everywhere, we have $\phi_{k} \rightarrow \rho^{2 q^{\prime}}$ almost everywhere. It then follows by Fatou's lemma that

$$
\int_{\Omega} u^{q} \rho^{2 q^{\prime}} w d x \leq c
$$

We deduce that $u \in L_{w, l o c}^{q}(\Omega)$. Since $\omega^{-\frac{q^{\prime}}{q}} \in L_{l o c}^{1}(\Omega)$, we obtain that $L_{l o c}^{1}(\Omega)$ by Hölder's inequality. If $u \in L_{w, l o c}^{q}(\Omega \backslash K) \cap u \in L^{1}(\Omega \backslash K)$ is a distributional solution of (1.22) in $\Omega \backslash K$, then $|u|$ is a nonnegative subsolution with the same integrability constraints and we derive $u \in L_{w, l o c}^{q}(\Omega) \cap L_{l o c}^{1}(\Omega)$.

If $\phi \in C_{0}^{\infty}(\Omega)$, we take $\phi\left(1-\zeta_{k}\right)^{2 q^{\prime}}$ for test function of equation (1.22) in $\mathcal{D}^{\prime}(\Omega \backslash K)$,

$$
-\int_{\Omega} u \Delta\left(\phi\left(1-\zeta_{k}\right)^{2 q^{\prime}}\right) d x+\int_{\Omega}|u|^{q-1} u \phi\left(1-\zeta_{k}\right)^{2 q^{\prime}} w d x=0 .
$$

Since $u \in L_{w, l o c}^{q}(\Omega), \phi$ has compact support, and $\zeta_{k} \rightarrow 0$ almost everywhere, we can pass to the limit as $k \rightarrow+\infty$ in the second integral using Lebesgue convergence theorem and obtain

$$
\int_{\Omega}|u|^{q-1} u \phi\left(1-\zeta_{k}\right)^{2 q^{\prime}} w d x \rightarrow \int_{\Omega}|u|^{q-1} u \phi w d x
$$

Moreover we can pass to the limit in the first integral expanding the laplacian. Using that $u \in L_{l o c}^{1}(\Omega)$ and that $\Delta \zeta_{k} \rightarrow 0$ in $L_{\omega}^{q^{\prime}}$, it is easy to prove from the previous computation that

$$
\int_{\Omega} u\left(1-\zeta_{k}\right)^{q^{\prime}} \Delta \phi d x \rightarrow \int_{\Omega} u \Delta \phi d x \quad \text { as } k \rightarrow \infty
$$

and

$$
\lim _{k \rightarrow \infty} \int_{\Omega} u\left(1-\zeta_{k}\right)^{2 q^{\prime}-1} \nabla \zeta_{k} . \nabla \phi d x=0=\lim _{k \rightarrow \infty} \int_{\Omega} u\left(1-\zeta_{k}\right)^{2 q^{\prime}-1} \phi \Delta \zeta_{k} d x .
$$

Hence

$$
-\int_{\Omega} u \Delta \phi d x+\int_{\Omega} u^{q} \phi w d x=0
$$

Step 2: The condition is necessary. Let $K$ be a compact set with positive $c_{q^{-}}^{\omega}$ capacity. According to [2][Th 2.5.3] there exists an extremal $\mu_{k} \in \mathfrak{M}_{b}^{+}(K)$ in the dual formulation (5.39) of the capacity. According to Theorem D, problem (5.16) 
with $\mu=\mu_{K}$ admits a positive solution which is therefore a positive solution of (5.35).

Aknowledgments The authors have been supported by the MathAmsud program 13Math-03 QUESP with fundings from CNRS, Ministère des Affaires Étrangères et Européennes, CONICET and MINCyT.

\section{References}

[1] D. Adams. Traces of potentials II. Indiana Univ. Math. J. 22, 907-918 (1973).

[2] D. Adams, L. Hedberg. Function spaces and potential theory. Grundleheren der Math. Wiss. 314, Springer-Verlag (1999).

[3] P. Baras, M. Pierre. Singularités éliminables pour des équations semi-linéaires. Ann. Inst. Fourier 34, 117-135 (1984).

[4] Ph. Benilan, H. Brezis. Nonlinear problems related to the Thomas-Fermi equation. Unpublished paper (1975). After Benilan's death a detailed version appeared in (2003), see the next reference.

[5] Ph. Benilan, H. Brezis. Nonlinear problems related to the Thomas-Fermi equation. Dedicated to Philippe Bénilan, J. Evol. Eq. 3, 673-770 (2003).

[6] Ph. Benilan, H. Brezis, M. Crandall. A semilinear equation in $L^{1}\left(\mathbb{R}^{N}\right)$. Ann. Sc. Norm. Sup. Pisa - Cl. di Scienze 2, 523-555 (1975).

[7] P. Billingsley. Convergence of probability measures. Second edition. Wiley Series in Probability and Statistics: Probability and Statistics. A Wiley-Interscience Publication. John Wiley \& Sons, Inc., New York (1999).

[8] H. Brezis, F. Browder. Strongly nonlinear elliptic boundary value problems. Ann. Sc. Norm. Sup. Pisa - Cl. di Scienze 5, 587-603 (1978).

[9] H. Brezis, M. Marcus, A. Ponce. Nonlinear Elliptic Equations with Measures Revisited. Ann. Math. Studies 163, 55-109, Princeton Univ. Press (2007).

[10] H. Brezis, A. Ponce. Kato's inequality when $\Delta u$ is a measure. C. R. Acad. Sci. Paris, Ser. I 338, 599-604, (2004).

[11] G. Dolzmann, N. Hungerbühler, S. Müller. Uniqueness and maximal regularity for nonlinear elliptic systems of n-Laplace type with measure valued right hand side. J. Reine Angew. Math. 520, 1-35 (2000). 
[12] E. B. Dynkin. Superdiffusion and Positive Solutions of Nonlinear Partial Differential Equations. Amer. Math. Soc., Providence, Rhode Island, Colloquium Publications 34, 2004.

[13] D. Feyel, A. de la Pradelle. Topologies fines et compactifications associées à certains espaces de Dirichlet. Ann. Inst. Fourier 27, 121-146 (1977).

[14] G. Grubb, Pseudo-differential boundary problems in $L_{p}$ spaces. Communications in Part. Diff. Equ. 15, 289-340 (1990).

[15] J. Heinonen, T. Kilpeleinen, O. Martio. Nonlinear Potential Theory of Degenerate Elliptic Equations. Dover Publishing Co (2006).

[16] M. Marcus, L. Véron. The boundary trace of positive solutions of semilinear elliptic equations: the subcritical case. Arch. Rat. Mech. Anal. 144, 201-231 (1998).

[17] M. Marcus, L. Véron. Capacitary estimates of positive solutions of semilinear elliptic equations with absorption. J. Europ. Math. Soc 6, 483-527 (2004).

[18] M. Marcus, L. Véron. Nonlinear second order Elliptic Equations involving measures. Series in Nonlinear Analysis and Applications 21, De Gruyter (2014).

[19] T. Miyakawa. On Morrey spaces of measures: basic properties and potential estimates. Hiroshima Math. J. 20, 213-220 (1990).

[20] V.G. Maz'ya. Sobolev spaces. Springer, Berlin,NewYork (1985).

[21] V.G. Maz'ya, I. Verbitsky. Capacitary inequalities for fractional integrals with applications to partial differential equations and Sobolev multipliers, Ark.Mat. 3, 81-115 (1995).

[22] B. Mselati. Classification and Probabilistic Representation of Positive Solutions of a Semilinear Elliptic Equations. Mem. Am. Math. Soc. 168, 2004.

[23] H. Triebel. Interpolation Theory, Function Spaces, Differential Operators. North-Holland Mathematical Library vol. 18, North-Holland (1978).

[24] H. Triebel. Theory of Function Spaces. Modern Birkhäuser Classics, Birkhäuser Verlag (1982).

[25] H. Triebel. Theory of Function Spaces II. Modern Birkhäuser Classics, Birkhäuser Verlag (1992).

[26] H. Triebel. Fractals and Spectra. Modern Birkhäuser Classics, Birkhäuser Verlag (1997). 
[27] J. L. Vazquez. On a semilinear equation in $\mathbb{R}^{2}$ involving bounded measures, Proc. Roy. Soc. Edinburgh 95A, 181-202 (1983).

[28] L. Véron. Singularities of solutions of second order quasilinear equations. Chapman and Hall/CRC Research Notes in Mathematics Series (1996).

[29] L. Véron. Elliptic equations involving measures. Stationary partial differential equations, Vol. I, 593-712, Handb. Differ. Equ., North-Holland, Amsterdam (2004).

[30] L. Véron, C. Yarur, Boundary value problems with measures for elliptic equations with singular potential, J. Functional Analysis, 262, 733-772, 2012.

[31] V.I. Yudovich. Some estimates connected with integral operators and with solutions of elliptic equations. Soviet Math. 7, 746-749 (1961). 